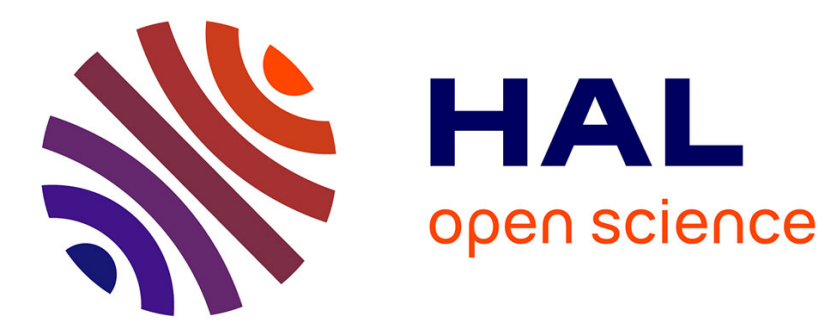

\title{
Output-feedback control of an underactuated network of interconnected hyperbolic PDE-ODE systems
}

\author{
Jeanne Redaud, Jean Auriol, Silviu-Iulian Niculescu
}

\section{To cite this version:}

Jeanne Redaud, Jean Auriol, Silviu-Iulian Niculescu. Output-feedback control of an underactuated network of interconnected hyperbolic PDE-ODE systems. Systems and Control Letters, 2021, 154, 10.1016/j.sysconle.2021.104984 . hal-03269701

HAL Id: hal-03269701

https://hal-centralesupelec.archives-ouvertes.fr/hal-03269701

Submitted on 24 Jun 2021

HAL is a multi-disciplinary open access archive for the deposit and dissemination of scientific research documents, whether they are published or not. The documents may come from teaching and research institutions in France or abroad, or from public or private research centers.
L'archive ouverte pluridisciplinaire HAL, est destinée au dépôt et à la diffusion de documents scientifiques de niveau recherche, publiés ou non, émanant des établissements d'enseignement et de recherche français ou étrangers, des laboratoires publics ou privés. 


\title{
Output-feedback control of an underactuated network of interconnected hyperbolic PDE-ODE systems
}

\author{
Jeanne Redaud ${ }^{2,1}$, Jean Auriol $^{1}$, Silviu-Iulian Niculescu ${ }^{2,1}$
}

\begin{abstract}
In this paper, the output feedback stabilization of an underactuated chain of scalar hyperbolic systems coupled at one end with a finite-dimensional system is addressed. As encountered in practical applications, only the first PDE subsystem is actuated. The measurements are collocated with the actuation. The proposed approach uses a recursive dynamics interconnection framework. More precisely, we first estimate (delayed) values of the states at each subsystem's boundaries. Then, we design a state-predictor that gives access to the boundary states' present and future values. It becomes finally possible to design a state-observer for the entire system. Combining this observer with a stabilizing state-feedback law, we can recursively design an output-feedback control law that stabilizes the whole chain. Some illustrative examples complete the presentation.
\end{abstract}

Keywords: backstepping, PDEs networks, time-delay systems, networks

\section{Introduction}

In this paper, we consider the problem of designing an output-feedback law for a chain of Partial Differential Equations (PDEs) coupled in its end with an Ordinary 5 Differential Equation (ODE). The actuator and the measurement are collocated at the end of the chain opposite to the ODE. Since only the first rightward convecting equation is actuated, the system can be said underactuated. PDEs are commonly used to model physical systems whom 10 dynamics depend on time and space variables. More specifically, networks of interconnected PDEs and ODEs are ${ }_{40}$ often used to represent complex phenomena, as thermoacoustic oscillations [1, traffic flows 2, oscillations along drilling pipes [3]. From an engineering point of view, inter15 connections between systems can be the source of multiple problems as instabilities or vibrations nodes that is preferable to avoid 4. However, stabilizing networked systems with good performance is not easy, especially when actuation is only available in discrete locations (usually at one 20 end of the system).

In the literature, various approaches have been developed ${ }_{50}$ to tackle similar issues. For instance, the well-known PI controller has been extended in [5] to a chain of linear hyperbolic systems. Flatness-based analysis is used to design 25 a state-feedback controller for hyperbolic PDEs networks in [6, 1, 8. The dynamics of characteristic lines are studied ${ }_{55}$ in [9] to design an output feedback control law for semilinear hyperbolic systems interconnected in series. Due to

\footnotetext{
${ }^{1}$ Université Paris-Saclay, CNRS, CentraleSupélec, Laboratoire des Signaux et Systèmes, 91190, Gif-sur-Yvette, France. Corre- 60 sponding author: jeanne.redaud@centralesupelec.fr

${ }^{2}$ INRIA
}

the delays inherent to such networks' structure, it is often necessary to design predictors to anticipate future values of the state [10].

Among these approaches, the backstepping method 11 has been extensively used to deal with interconnected PDEs. Thanks to it, some complex problems have been solved during the last decade: stabilization of wave PDEs with nonlocal terms 12, and of multiple-dimensional PDEs, such as 2D Navier-Stokes equations [13; or boundary feedback controller design for more or less complex hyperbolic systems [14, 15]. Finally, it is worth mentioning that the general problem of stabilizing an arbitrary number of interconnected scalar PDEs has been solved in [16]. The proposed approach was based on a single backstepping transformation (the observer being designed through a dual approach). However, it required solving a complex set of kernel equations and could not be easily extended to more complex systems (since new target systems would be needed). The backstepping approach has also been used to stabilize PDEs interconnected with ODEs in chain structures and, in particular, to design control laws stabilizing an ODE-PDE-ODE structure [17, 18, 19, 20, 21, and PDE-ODE-PDE structures 22, 23].

More recently, this approach has been implemented in 24] to delay-robustly stabilize a system of hyperbolic PDEs coupled with an ODE in an $n+m$ PDE-ODE structure. A chain of PDEs coupled at one end with an ODE is of high interest for industrial applications. Indeed, the dynamics of finite-dimension actuators or loads at one end of the system can be modeled by an ODE. For example, the dynamics of the extremity of a drilling pipe, called the Bottom Hole Assembly (BHA), can be modeled by an ODE subsystem at the end of a chain of interconnected 


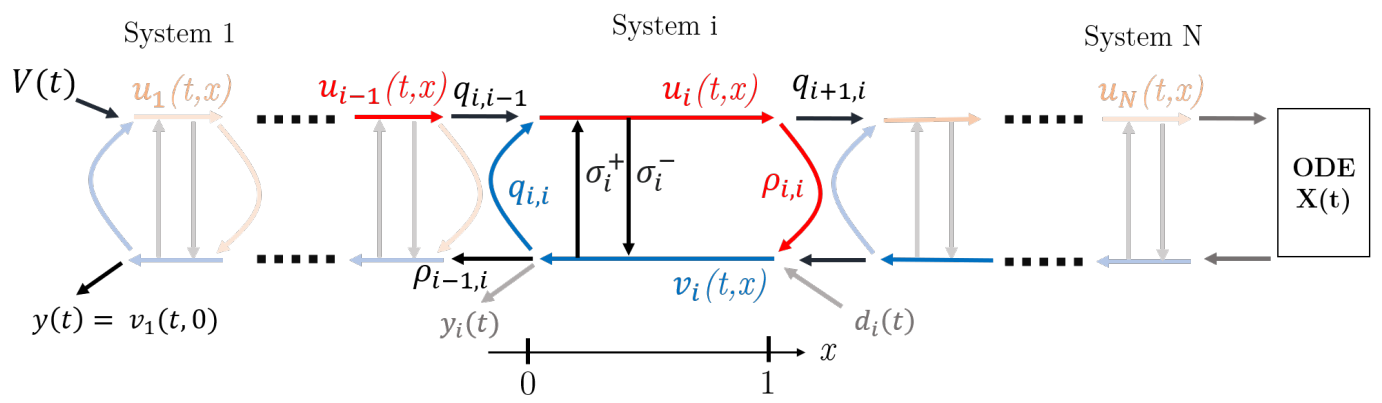

Figure 1: Schematic representation of the system (1)- 5 .

PDEs (propagation along the pipe).

However, since the backstepping transformations must be105 specifically designed for each system, it lacks adaptability from one network to another. For instance, adding one more PDE subsystem in a chain structure was not possible. To remedy this disadvantage, a new recursive dynamics interconnection framework was recently devel-110 oped in [25]. The main idea of this new framework is to explo the interconnection structure by recursively designing observers or controllers for each subsystem, gathering information on the nodes of the networks, and, finally, rewriting each subsystem in the form of transport equa-115 tions. Interconnections properties are used to recursively

75 build a state-feedback control law, making each subsystem's output converging towards some virtual control law that will act on the next downstream subsystem, taking into account the counter effect of this very downstream 120 subsystem as a virtual perturbation. In that sense, such ${ }_{80}$ an approach presents some similarities with the flatnessbased feedforward tracking control design developed in [8]. Though, a limitation of 25] was that all the boundary states were assumed to be available to design the control law. In this paper, we solely use a measure collocated 85 with the actuator at one extremity of the chain opposite the ODE. We then design an observer for the system using this single measure. In our opinion, this step is necessary to develop operating methods implemented on real systems. More precisely, this paper's main contribution ${ }_{90}$ is to estimate the chain's whole state using the available measurement. This yields to the design of an appropriate output feedback control law.

Our approach is based on a recursive dynamics framework and is described as follows. We first use elementary invertible backstepping transforms and each subsystem's transport structure to recursively estimate the boundary states. Due to the physical system's natural inertia, our estimation is only available with a specific delay. Following some of the ideas developed for finite-dimensional systems (see, for instance, [26]), we then design a predictor to estimate the state values in real-time and also in the future. Inspired by the results from [25], we finally design an output feedback control law for the chain of hyperbolic systems coupled with an ODE at its one end.

To the best of the authors' knowledge, such a paper represents a novelty in the literature. Unlike in 16, the chain of PDEs considered in this paper is coupled with an ODE. Moreover, the recursive dynamics interconnection framework proposed herein is more generic and more comfortable to implement.

The layout of the paper is as follows: in Section 2, we describe the system under consideration and describe our strategy. Then the boundary state estimation is presented in Section 3. Next, in Section 4, using these estimations, we design a boundary-states' predictor that we extend to the entire state of each subsystem. In Section 5, we derive from 25] an output-feedback control law, whom efficiency is illustrated by simulation results and an engineering example in section 6. Finally, some concluding remarks (Section 7 end the paper.

\section{Problem description}

\subsection{System under consideration}

We consider a system composed of $N>0$ interconnected PDE systems in a chain structure, coupled with an ODE at the end of the chain. This system is schematically represented on Figure 1. Each PDE subsystem corresponds to a linear hyperbolic system of two coupled scalar equations. The PDEs subsystems are modeled by the set of equations $(i \in \llbracket 1, N \rrbracket)$ :

$$
\begin{aligned}
& \partial_{t} u_{i}(t, x)+\lambda_{i} \partial_{x} u_{i}(t, x)=\sigma_{i}^{+}(x) v_{i}(t, x), \\
& \partial_{t} v_{i}(t, x)-\mu_{i} \partial_{x} v_{i}(t, x)=\sigma_{i}^{-}(x) u_{i}(t, x) .
\end{aligned}
$$

We assume that the state variables have been normalized, such that $t>0, x \in[0,1]$. The in-domain coupling terms $\sigma_{i}^{+}$and $\sigma_{i}^{-}$are continuous functions. Note that our results could be extended to spatially-varying $\mathcal{C}^{1}$ transport velocities $\lambda_{i}>0, \mu_{i}>0$. The initial conditions associated to equations (1) and (2) are denoted $u_{i}^{0}(\cdot)=u_{i}(0, \cdot)$ and $v_{i}^{0}(\cdot)=v_{i}(0, \cdot)$ and are defined in $L^{2}([0,1], \mathbb{R})$. The different subsystems are connected through their boundaries. More precisely, we have the boundary conditions:

$$
u_{i}(t, 0)=q_{i, i} v_{i}(t, 0)+q_{i-1, i} u_{i-1}(t, 1)+\delta_{1}^{i} V(t),
$$




$$
v_{i}(t, 1)=\rho_{i, i} u_{i}(t, 1)+\rho_{i, i+1} v_{i+1}(t, 0)+\delta_{N}^{i} C X(t),
$$

where the different couplings $q_{i, j}$ and $\rho_{i, j}$ are constant and where $V(t)$ is the real-valued control input we want to design. In order to keep equations (3)-(4) in the most general ${ }_{155}$ form, we used the convention $q_{0,1}=0$ and $\rho_{N, N+1}=0$. The notation $\delta_{j}^{i}$ stands for the Kronecker symbol $\left(\delta_{i}^{j}=1\right.$ if and only if $i=j)$. The last PDE subsystem $\left(N^{t h}\right.$ subsystem) is coupled with an ODE of dimension $p \in \mathbb{N}$ such that:

$$
\dot{X}(t)=A X(t)+B u_{N}(t, 1),
$$

with $A \in \mathbb{R}^{p \times p}, B \in \mathbb{R}^{p \times 1}, C \in \mathbb{R}^{1 \times p}$ constant matrices. The corresponding initial condition $X_{0}=X(0)$ belongs to $\mathbb{R}^{p}$ ( $p$ being a positive integer). The last subsystem can model, for instance, a heavy element at the end of the drill-string, regarded as a mass-spring-damper system, which is well described by an ODE. The system (1)-(5) is said to be under-actuated, since only the first subsystem is forming change of variables to rewrite the network as a single PDE-ODE system where the PDE is composed of $N$ leftward convecting equations and $N$ rightward convecting ones. As only one of the rightward convecting equathe equations propagating in one direction are actuated), the system can be said underactuated. We will use the interconnections through the different subsystems' boundaries to design an observer and act from one system to the next one. We assume that we only measure the first subsystem's state $y(t)=v_{1}(t, 0)$. The well-posedness of the system (1)-(5) in the sense of the $L^{2}$-norm is guaranteed by [28, Appendix A].

\subsection{Problem formulation}

Denote by $u$ (resp. $v$ ) the concatenation of the states $u_{i}$ (resp. $v_{i}$ ), let us define the $\Xi$-norm ${ }^{3}$ of the state $(u, v, X)$ as $\|(u, v, X)\|_{\Xi}=\left(\sum_{i=1}^{N}\left(\left\|u_{i}\right\|_{L^{2}}^{2}+\left\|v_{i}\right\|_{L^{2}}^{2}\right)+\|X\|_{\mathbb{R}^{p}}^{2}\right)^{\frac{1}{2}}$. The objective of this paper is to design an output control ${ }_{190}$ law $V(t)$ that exponentially stabilizes the system (1)-(5) in the sense of the $\Xi$-norm. More precisely, we want to design a control law such that $\exists \nu>0, C_{0}>0, \forall\left(u_{0}, v_{0}, X_{0}\right) \in$ $L^{2}([0,1])^{n+m} \times \mathbb{R}^{p}$, all solutions of the closed-loop (1)-(5) satisfy

$$
\|(u, v, X)\|_{\Xi} \leq C_{0} \mathrm{e}^{-\nu t}\left\|\left(u_{0}, v_{0}, X_{0}\right)\right\|_{\Xi} .
$$

A stabilizing state-feedback control law has already been designed in 25] for the class of system under consideration. The proposed control law was based on a recursive interconnected dynamics framework. Roughly speaking, such a control law was recursively obtained by considering stabilizing virtual inputs for each subsystem and

${ }^{3}$ This norm represents the sum of the square of the $L^{2}$-norm of each PDE state and the Euclidean norm of the ODE state. ensuring that each subsystem's output converges to the corresponding desired virtual input. This new framework allows for a "plug-and-play"-like approach to control design since additional subsystems satisfying similar conditions can be added to the network using the same procedure. More precisely, for each subsystem, we propose a flatness-based feedforward tracking control design [29, 7] so that the output of the considered subsystem tracks a function that stabilizes the downstream subsystems. This approach requires predicting future values of the PDEs and the ODE states. The proposed predictors require the knowledge of the state at different points of the domain. In this paper, we adapt the recursive dynamics interconnected framework from 25 to design an output feedback control law. We first reconstruct the boundary states (i.e., the state's values at the connections between the different subsystems) using the available measurement.

Consider now Figure 1. It can be seen that a system $i$ will act on the downstream subsystem $i+1$ through $u_{i}(t, 1)$, and on the upstream subsystem $i-1$ through $v_{i}(t, 0)$. Then, going recursively from one subsystem to the next, we can estimate the states at each subsystem's boundaries. However, due to the system's natural inertia (induced by the transport phenomenon in the PDEs), there are some delays in the estimations. More precisely, we can only estimate past values of the boundary states. However, it is then possible to apply the predictors designed in 25] to obtain non-delayed estimations. Such an approach (that combines delayed estimations and state-predictions) is similar to the one used for finite-dimensional systems 30. As the control law designed in 25] requires future values of the state, we predict the boundary states' values on a larger time horizon. We can then adapt the results to stabilize the whole chain with an output-feedback law. Note one developed in 16 for a chain of scalar PDE systems. Indeed, it involves a specific backstepping transformation and, in our opinion, it is hard to generalize to different types of chains. Conversely, we believe that the recursive framework introduced in this paper offers interesting perspectives, and it can be applied to different classes of interconnected systems.

\subsection{Structural assumptions}

The design of a state observer and a stabilizing feedback law requires several necessary and non-restrictive assumptions. First, to guarantee the observability of the whole system, we need an assumption regarding the observability of the ODE system coupled with the $N^{t h}$ subsystem at the end of the chain:

Assumption 1. The ODE-state $X$ is detectable, that is: $\exists L \in \mathbb{R}^{p \times 1}, A+L C$ is Hurwitz.

Next, to estimate the states of the downstream subsystems using the measurement from the upstream subsystem; we need the following natural assumption: 


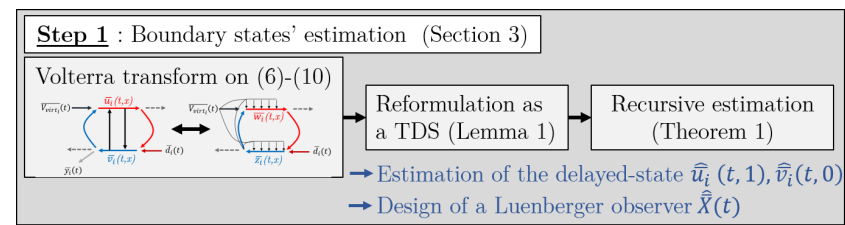

$\underline{\text { Step } 2}$ : Boundary states' prediction (Section 4)

Volterra transform on (6)-(10)

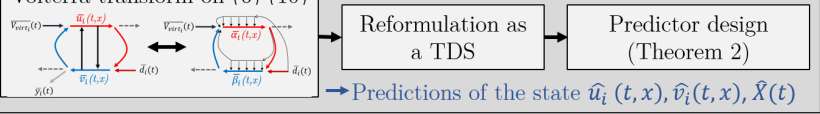

\begin{tabular}{|c|c|c|c|}
\hline \multicolumn{3}{|c|}{ Step 3 : Output-feedback control law design (Section 5) } & \\
\hline $\begin{array}{c}\text { Stabilizing control } \\
\text { law (without pert.) } \\
\text { (property 3) }\end{array}$ & $\begin{array}{c}\text { Control law for } \\
\text { output tracking } \\
\text { (property 4) }\end{array}$ & $\begin{array}{l}\text { Recursive design } \\
\text { (Theorem 3) }\end{array}$ & \\
\hline
\end{tabular}

Assumption 2. We have for all $i \in \llbracket 2, N \rrbracket, \rho_{i-1, i} \neq 0$.

Then, we need to guarantee the whole system to be stabi- ${ }^{255}$ lizable. Thus, we will have:

Assumption 3. The ODE-state $X$ is stabilizable, that is: $\exists K \in \mathbb{R}^{1 \times p}, A+B K$ is Hurwitz.

To stabilize the downstream subsystems' states using actuation from the upstream subsystem, we need the following natural assumption:

Assumption 4. We have for all $i \in \llbracket 2, N \rrbracket, q_{i-1, i} \neq 0$.

To these assumptions, we add the following condition:

Assumption 5. The open loop system (1)-(5) (i.e. $V(t) \equiv$ 0 ) in the absence of in-domain coupling terms (i.e. $\sigma_{i} \equiv$ 0 ) and of the $O D E$ (i.e $X \equiv 0$ ) is exponentially stable (in the sense of the $L^{2}$ norm).

It has been shown in 31 that a necessary condition to guarantee the existence of robustness margins for an arbitrary closed-loop system is that the open-loop transfer function must have a finite number of poles on the closed right half-plane. For the system (1)-(5), 24, 32 proved that this implies Assumption 5. Some explicit conditions to verify such an assumption can be found in [33, 32, 16. Consequently, Assumption 5 is not restrictive as it is necessary for the existence of robustness margins for the closedloop system. For a deeper discussion of the sensitivity with respect to the delay parameter, the reader is referred to 34 .

\subsection{Description of the strategy}

We give a here an overview of the proposed strategy (which is summarized in Figure 2)

- First, due to the system's transport structure, we estimate delayed values of the state $\left(\bar{u}_{i}, \bar{v}_{i}\right)$. We use a first invertible backstepping transform (between 0 and $x$ ), to map the initial PDE subsystem to a target subsystem $\left(\bar{w}_{i}, \bar{z}_{i}\right)$. We rewrite it as a time-delay system. We can then estimate delayed-values of the boundary PDE states (under Assumption 2) and of the ODE state (Assumption 1), using a classical Luenberger observer.

- similarly to what is done for finite-dimensional systems [30, we then design a state-predictor to have access to real-time estimations. We use a second invertible backstepping transform (between $x$ and 1), to map the initial PDE subsystem to a target subsystem $\left(\bar{\alpha}_{i}, \bar{\beta}_{i}\right)$. We rewrite the boundary states as a time-delay system and use this formulation to design the boundary states' predictor.

- finally, following [25], we use the predictors computed using the estimations to design a causal output feedback control law $V(t)$ that exponentially stabilizes the entire chain (under Assumption 3 and Assumption 4.

\section{Boundary state estimation}

In this section, we estimate the values of the boundary PDEs states using the available measurement. Due to the transport delay involved by each PDE system, these estimations correspond to past values of the boundary states. The time ahead which an estimation of a boundary state is available depends on the transport velocities $\lambda_{i}, \mu_{i}$.

\subsection{Time-delay system}

To simplify the computations and inspired by [30, we directly consider the delayed states of our system. Let us consider $\tau>0$ a fixed, known delay, whose value will be given later. We define the $\tau$-delay operator ${ }^{-}$, such that: $\forall \gamma \in L^{2}([0,+\infty[, \mathbb{R}), \forall t>\tau, \bar{\gamma}(t)=\gamma(t-\tau)$. Using this operator, we can rewrite the system (1)-(5). Thus, for all $t \geq \tau$, we have:

$$
\begin{aligned}
& \partial_{t} \bar{u}_{i}(t, x)+\lambda_{i} \partial_{x} \bar{u}_{i}(t, x)=\sigma_{i}^{+}(x) \bar{v}_{i}(t, x), \\
& \partial_{t} \bar{v}_{i}(t, x)-\mu_{i} \partial_{x} \bar{v}_{i}(t, x)=\sigma_{i}^{-}(x) \bar{u}_{i}(t, x), \\
& \dot{\bar{X}}(t)=A \bar{X}(t)+B \bar{u}_{N}(t, 1),
\end{aligned}
$$

along with the boundary conditions :

$$
\begin{aligned}
& \bar{u}_{i}(t, 0)=q_{i, i} \bar{v}_{i}(t, 0)+q_{i-1, i} \bar{u}_{i-1}(t, 1), \\
& \bar{v}_{i}(t, 1)=\rho_{i, i} \bar{u}_{i}(t, 1)+\rho_{i, i+1} \bar{v}_{i+1}(t, 0) .
\end{aligned}
$$

Note that to simplify the notations and avoid any useless case distinction, we now denote $q_{1,0}=1, \bar{u}_{0}(t, 1) \doteq \bar{V}(t)$, $\rho_{N, N+1}=C$ and $\bar{v}_{N+1}(t, 0)=\bar{X}(t)$. For this time-delay system, the available measurement is now defined as $\bar{y}(t)=$ $y(t-\tau)$. Thus, it means that we know $\tau$-ahead future values of $\bar{y}$. Consider now an isolated subsystem $i$ as illustrated in Figure 3. For this subsystem, we define the 
delay $\tau_{i}$ inherent to the dynamics of the upstream subsystems as $\tau_{i}=\sum_{j=1}^{i-1} \frac{1}{\mu_{j}}$. For any subsystem $i$, we assume we have access to a virtual measure $\bar{y}_{i}$ defined as:

$$
\bar{y}_{i}(t)=v_{i}\left(t-\tau_{i}, 0\right)=\bar{v}_{i}\left(t+\tau-\tau_{i}, 0\right) .
$$

This definition is causal as it only requires past values of the state $\bar{v}_{i}$. Obviously, the term virtual measure means that we should be able to estimate $\bar{y}_{i}$ at some point. Note that $\bar{y}_{1}=\bar{y}$, which is known on the time interval $[t, t+\tau]$. Our isolated subsystem $i$ is also subject to a disturbance $d_{i}(t)$ that corresponds to the downstream subsystem's action. It is defined as $\bar{d}_{i}(t)=\rho_{i, i+1} \bar{v}_{i+1}(t, 0)$

For any function $\gamma$, we denote $\hat{\gamma}$ the corresponding $o b$ server state (or estimated state). In this section, we show that we can design an observer $\hat{\bar{u}}_{i}(t, 1), \hat{\bar{v}}_{i}(t, 0)$ for the de- ${ }_{31}$ layed boundary states of each subsystem. More precisely, we prove the following property:

Property 1. Observability of the boundary states: For all $i \in \llbracket 1, N \rrbracket$, there exists two functions $\hat{u}_{i}(., 1), \hat{v}_{i}(., 0)$, that causally depend on the measurement $y(t)$ and on the control law $V(t)$ such that: $\left\|\bar{u}_{i}(t, 1)-\hat{\bar{u}}_{i}(t, 1)\right\|_{L^{2}} \underset{t \rightarrow+\infty}{\longrightarrow} 0$ and $\left\|\bar{v}_{i}(t, 0)-\hat{\bar{v}}_{i}(t, 0)\right\|_{L^{2}} \underset{t \rightarrow+\infty}{\longrightarrow} 0$. Moreover, there exists $\dot{X}$ that causally depends on the measurement $y(t)$ and on the control law $V(t)$ such that: $\|\bar{X}(t)-\hat{\bar{X}}(t)\| \underset{t \rightarrow+\infty}{\longrightarrow} 0$

Remark 1. As it will be further highlighted, we can get a stronger result since the proposed estimation procedure will ${ }_{315}$ ary states. However, we will use the notation . to emphasize that these boundary states are not supposedly known but computed from the measurement $y(t)$.

Our strategy to show Property 1 can be resumed as follows:

- first, using an invertible backstepping transform to move the in-domain couplings to the right boundary of any subsystem $i$. It is important to point out that this transformation does not modify the virtual measurement;

- second, using estimation (or knowledge) of the upstream boundary state $\bar{u}_{i-1}(t, 1)$ and the virtual measurement $\bar{y}_{i}(t)$ to estimate the disturbance term $\bar{d}_{i}(t)$, and the action on the downstream subsystem $\bar{u}_{i}(t, 1)$;

- finally, estimating recursively the boundary states $\bar{v}_{i}(t, 0)$ and $\bar{u}_{i}(t, 1), \forall i \in \llbracket 1, N \rrbracket$.

With the observations above, consider a subsystem $i(i \in$ $\llbracket 1, N \rrbracket)$, as illustrated in Figure 3 . We assume that we have access to the virtual measurement $\bar{y}_{i}(t)=\bar{v}_{i}(t+\tau-$ $\left.\tau_{i}, 0\right)$, and to the action of the upstream subsystem $\bar{u}_{i-1}(t, 1)$ on a time interval $\left[t, t+\tau-\tau_{i}\right]$. Note that this assumption is obviously satisfied for $i=1$ since $\tau_{1}=0$. The following lemma assesses that the right output of our considered subsystem (namely $\bar{u}_{i}(t, 1)$ ) and the disturbance

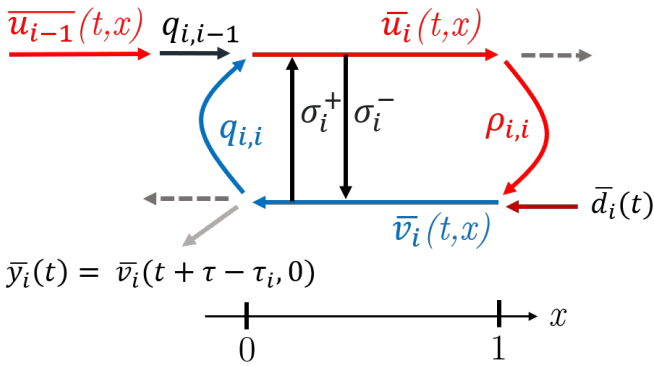

Figure 3: Schematic representation of one subsystem i.

input $\bar{d}_{i}(t)=\rho_{i, i+1} \bar{v}_{i+1}(t, 0)$ can be expressed as linear functions of the virtual measurement and of the left input $\bar{u}_{i-1}(t, 1)$.

Lemma 1. Consider $i \in \llbracket 1, N \rrbracket$ and assume that $\bar{u}_{i-1}$ is known on the time interval $\left[t, t+\frac{1}{\mu_{i}}\right]$ and that the function $\bar{y}_{i}$ is available. Then, there exist two linear operators $\mathcal{L}_{u_{i}}$ and $\mathcal{L}_{d_{i}}$, such that for $t>\tau+\frac{1}{\lambda_{i}}$,

$$
\begin{aligned}
\bar{u}_{i}(\nu, 1) & =\mathcal{L}_{u_{i}}\left(\bar{y}_{i}(\cdot), \bar{u}_{i-1}(\cdot, 1)\right), \\
\bar{d}_{i}(\nu) & =\mathcal{L}_{d_{i}}\left(\bar{y}_{i}(\cdot), \bar{u}_{i-1}(\cdot, 1)\right),
\end{aligned}
$$

for all $\nu \in\left[t, t+\tau-\tau_{i+1}\right]$.

To prove Lemma 1, we will use a backstepping approach inspired by [27, 14, 35] coupled with a neutral-type formulation. The next two subsections describe the explicit steps of the proof in a constructive way.

\subsection{Backstepping transform}

Consider the $i^{\text {th }}$ subsystem $(i \in \llbracket 1, N \rrbracket)$, as represented in Figure 3. We first apply the invertible Volterra transform:

$$
\begin{aligned}
\bar{u}_{i}(t, x)= & \bar{w}_{i}(t, x) \\
& -\int_{0}^{x} L_{i}^{w w}(x, \xi) \bar{w}_{i}(t, \xi)+L_{i}^{w z}(x, \xi) \bar{z}_{i}(t, \xi) \mathrm{d} \xi \\
\bar{v}_{i}(t, x)= & \bar{z}_{i}(t, x) \\
& -\int_{0}^{x} L_{i}^{z w}(x, \xi) \bar{w}_{i}(t, \xi)+L_{i}^{z z}(x, \xi) \bar{z}_{i}(t, \xi) \mathrm{d} \xi
\end{aligned}
$$

where the kernels $L^{*}$ are continuous functions defined on the lower triangular domain $\{(x, \xi) \mid 0 \leq x \leq 1,0 \leq \xi \leq$ $x\}$. They satisfy the following set of equations

$$
\begin{aligned}
& \lambda_{i} \partial_{x} L_{i}^{w w}(x, \xi)+\lambda_{i} \partial_{\xi} L_{i}^{w w}(x, \xi)=\sigma_{i}^{+} L_{i}^{z w}(x, \xi), \\
& \lambda_{i} \partial_{x} L_{i}^{w z}(x, \xi)-\mu_{i} \partial_{\xi} L_{i}^{w z}(x, \xi)=\sigma_{i}^{+} L_{i}^{z z}(x, \xi), \\
& \mu_{i} \partial_{x} L_{i}^{z w}(x, \xi)-\lambda_{i} \partial_{\xi} L_{i}^{z w}(x, \xi)=-\sigma_{i}^{-} L_{i}^{w w}(x, \xi), \\
& \mu_{i} \partial_{x} L_{i}^{z z}(x, \xi)+\mu_{i} \partial_{\xi} L_{i}^{z z}(x, \xi)=-\sigma_{i}^{-} L_{i}^{w z}(x, \xi),
\end{aligned}
$$

with the boundary conditions:

$$
L_{i}^{z w}(x, x)=\frac{\sigma_{i}^{-}}{\lambda_{i}+\mu_{i}}, L_{i}^{w z}(x, x)=-\frac{\sigma_{i}^{+}}{\lambda_{i}+\mu_{i}}
$$


In order for the problem to be well-posed, we add two arbitrary boundary conditions : $\forall \xi \in[0,1] L_{i}^{z z}(1, \xi)=$ $\rho_{i, i} L_{i}^{w z}(1, \xi)$ and $\forall \xi \in[0,1] L_{i}^{w w}(1, \xi)=0$. According to 14,35 , this system admits a unique solution.

With this transformation, we can map the original system (6)- 10 to the target system :

$$
\begin{aligned}
\partial_{t} \bar{w}_{i}(t, x)+\lambda_{i} \partial_{x} \bar{w}_{i}(t, x) & =f_{i}(x) \bar{v}_{i}(t, 0) \\
& +h_{i}(x) \bar{u}_{i-1}(t, 1), \\
\partial_{t} \bar{z}_{i}(t, x)-\mu_{i} \partial_{x} \bar{z}_{i}(t, x) & =g_{i}(x) \bar{v}_{i}(t, 0) \\
& +k_{i}(x) \bar{u}_{i-1}(t, 1),
\end{aligned}
$$

with the following boundary conditions

$$
\begin{aligned}
\bar{w}_{i}(t, 0) & =q_{i, i-1} \bar{u}_{i-1}(t, 1)+q_{i, i} \bar{v}_{i}(t, 0), \\
\bar{z}_{i}(t, 1) & =\rho_{i, i} \bar{u}_{i}(t, 1)+\bar{d}_{i}(t) \\
& +\int_{0}^{1}\left(L_{i}^{z w}(1, \xi) \bar{w}_{i}(t, \xi)+L_{i}^{z z}(1, \xi) \bar{z}_{i}(t, \xi)\right) \mathrm{d} \xi .
\end{aligned}
$$

Note that in-domain couplings have been moved to the right boundary of our subsystem $(x=1)$. The functions $f_{i}, g_{i}, h_{i}, k_{i}$ are real-valued functions defined on $[0,1]$. They satisfy the integral equations:

$$
\begin{aligned}
f_{i}(x)- & \int_{0}^{x} L_{i}^{w w}(x, \xi) f_{i}(\xi)+L_{i}^{w z}(x, \xi) g_{i}(\xi) \mathrm{d} \xi \\
& =\lambda_{i} q_{i, i} L_{i}^{w w}(x, 0)-\mu_{i} L_{i}^{w z}(x, 0), \\
g_{i}(x)- & \int_{0}^{x} L_{i}^{z w}(x, \xi) f_{i}(\xi)+L_{i}^{z z}(x, \xi) g_{i}(\xi) \mathrm{d} \xi \\
& =\lambda_{i} q_{i, i} L_{i}^{z w}(x, 0)-\mu_{i} L_{i}^{z z}(x, 0), \\
h_{i}(x) & -\int_{0}^{x} L_{i}^{w w}(x, \xi) h_{i}(\xi)+L_{i}^{w z}(x, \xi) k_{i}(\xi) \mathrm{d} \xi \\
& =\lambda_{i} q_{i, i-1} L_{i}^{w w}(x, 0), \\
k_{i}(x) & -\int_{0}^{x} L_{i}^{z w}(x, \xi) h_{i}(\xi)+L_{i}^{z z}(x, \xi) k_{i}(\xi) \mathrm{d} \xi \\
& =\lambda_{i} q_{i, i-1} L_{i}^{z w}(x, 0),
\end{aligned}
$$

The equations 22)-(25) are Volterra equations and, according to 36, admit a unique solution. Note that we have chosen to preserve the terms $\bar{u}_{i}(t, 1)$ and $\bar{v}_{i}(t, 0)$ in the target system (18)-(21) instead of replacing them by $\bar{z}_{i}$ and $\bar{w}_{i}$. This will simplify the estimation procedure.

\subsection{Neutral-type formulation of the boundary states}

In order to find the linear operators $\mathcal{L}_{u_{i}}, \mathcal{L}_{d_{i}}$, we now rewrite the new system (18)-21) as a functional differential equation of neutral type. Using the method of characteristics, we have, $\forall x \in[0,1], \forall t>\frac{1}{\mu_{i}}+\frac{1}{\lambda_{i}}$ :

$$
\begin{gathered}
\bar{w}_{i}(t, x)=\bar{w}_{i}\left(t-\frac{x}{\lambda_{i}}, 0\right)+\int_{0}^{\frac{x}{\lambda_{i}}} f_{i}\left(x-\lambda_{i} s\right) \bar{v}_{i}(t-s, 0) \\
+h_{i}\left(x-\lambda_{i} s\right) \bar{u}_{i-1}(t-s, 1) \mathrm{d} s \\
\bar{z}_{i}(t, x)=\bar{z}_{i}\left(t-\frac{1-x}{\mu_{i}}, 1\right)+\int_{0}^{\frac{1-x}{\mu_{i}}} g_{i}\left(x+\mu_{i} s\right) \bar{v}_{i}(t-s, 0)^{325}
\end{gathered}
$$

$$
+k_{i}\left(x+\mu_{i} s\right) \bar{u}_{i-1}(t-s, 1) \mathrm{d} s .
$$

Consequently, we obtain:

$$
\begin{aligned}
\bar{u}_{i}(t, 1)= & \bar{w}_{i}(t, 1)-\int_{0}^{1} L_{i}^{w z}(1, \xi) \bar{z}_{i}(t, \xi) \mathrm{d} \xi \\
& \quad \text { from }[1] \text { and } L_{i}^{w w}(1, \xi)=0 \forall \xi \in[0,1] \\
= & q_{i, i-1} \bar{u}_{i-1}\left(t-\frac{1}{\lambda_{i}}, 1\right)+q_{i, i} \bar{v}_{i}\left(t-\frac{1}{\lambda_{i}}, 0\right) \\
+ & \int_{0}^{\frac{1}{\lambda_{i}}} \bar{u}_{i-1}(t-s, 1) \mathcal{U}_{i}^{-}(s)+\bar{v}_{i}(t-s, 0) \mathcal{V}_{i}^{-}(s) \mathrm{d} s \\
+ & \quad \int_{0}^{\frac{1}{\mu_{i}}} \bar{u}_{i-1}(t+s, 1) \mathcal{U}_{i}^{+}(s)+\bar{v}_{i}(t+s, 0) \mathcal{V}_{i}^{+}(s) \mathrm{d} s \\
& \quad \operatorname{from~} 26] \\
= & q_{i, i-1} \bar{u}_{i-1}\left(t-\frac{1}{\lambda_{i}}, 1\right)+q_{i, i} \bar{y}_{i}\left(t-\frac{1}{\lambda_{i}}-\tau+\tau_{i}\right) \\
+ & \int_{0}^{\frac{1}{\lambda_{i}}} \bar{u}_{i-1}(t-s, 1) \mathcal{U}_{i}^{-}(s)+\bar{y}_{i}\left(t-s-\tau+\tau_{i}\right) \mathcal{V}_{i}^{-}(s) \mathrm{d} s \\
+ & \int_{0}^{\frac{1}{\mu_{i}}} \bar{u}_{i-1}(t+s, 1) \mathcal{U}_{i}^{+}(s)+\bar{y}_{i}\left(t+s-\tau+\tau_{i}\right) \mathcal{V}_{i}^{+}(s) \mathrm{d} s
\end{aligned}
$$

where for all $s \in\left[0, \frac{1}{\lambda_{i}}\right]$

$$
\left\{\begin{array}{l}
\mathcal{U}_{i}^{-}(s)=h_{i}\left(1-\lambda_{i} s\right) \\
\mathcal{V}_{i}^{-}(s)=f_{i}\left(1-\lambda_{i} s\right)
\end{array}\right.
$$

and where for all $s \in\left[0, \frac{1}{\mu_{i}}\right]$

$$
\left\{\begin{aligned}
\mathcal{U}_{i}^{+}(s) & =\mu_{i} \int_{s}^{\frac{1}{\mu_{i}}} L_{i}^{w z}\left(1, \mu_{i} \nu\right) k_{i}\left(\mu_{i}(\nu-s)\right) \mathrm{d} \nu \\
\mathcal{V}_{i}^{+}(s) & =\mu_{i}\left(-L_{i}^{w z}\left(1, \mu_{i} s\right)\right. \\
& \left.+\int_{s}^{\frac{1}{\mu_{i}}} L_{i}^{w z}\left(1, \mu_{i} \nu\right) g_{i}\left(\mu_{i}(\nu-s)\right) \mathrm{d} \nu\right)
\end{aligned}\right.
$$

Similarly, we obtain

$$
\begin{gathered}
\bar{d}_{i}(t)=\bar{v}_{i}(t, 1)-\rho_{i, i} \bar{u}_{i}(t, 1) \quad \text { by definition } \\
=\bar{v}_{i}\left(t+\frac{1}{\mu_{i}}, 0\right)-\int_{0}^{\frac{1}{\mu_{i}}} g_{i}\left(\mu_{i} s\right) \bar{v}_{i}\left(t+\frac{1}{\mu_{i}}-s, 0\right) \\
\quad+k_{i}\left(\mu_{i} s\right) \bar{u}_{i-1}\left(t+\frac{1}{\mu_{i}}-s, 1\right) \mathrm{d} s \\
-\rho_{i, i} q_{i, i-1} \bar{u}_{i-1}\left(t-\frac{1}{\lambda_{i}}, 1\right)-\rho_{i, i} q_{i, i} \bar{v}_{i}\left(t-\frac{1}{\lambda_{i}}, 0\right) \\
-\int_{0}^{\frac{1}{\lambda_{i}}} \bar{v}_{i}(t-s, 0) \mathcal{I}_{v_{i}}(s)+\bar{u}_{i-1}(t-s, 1) \mathcal{I}_{u_{i}}(s) \mathrm{d} s \\
=\bar{y}_{i}\left(t-\tau+\tau_{i}+\frac{1}{\mu_{i}}\right)-\rho_{i, i} q_{i, i-1} \bar{u}_{i-1}\left(t-\frac{1}{\lambda_{i}}, 1\right) \\
-\rho_{i, i} q_{i, i} \bar{y}_{i}\left(t-\tau+\tau_{i}-\frac{1}{\lambda_{i}}\right) \\
-\int_{0}^{\frac{1}{\mu_{i}}} g_{i}\left(\mu_{i} s\right) \bar{y}_{i}\left(t-\tau+\tau_{i}+\frac{1}{\mu_{i}}-s\right) \\
\quad+k_{i}\left(\mu_{i} s\right) \bar{u}_{i-1}\left(t+\frac{1}{\mu_{i}}-s, 1\right) \mathrm{d} s \\
-\int_{0}^{\frac{1}{\lambda_{i}}} \bar{y}_{i}\left(t-\tau+\tau_{i}-s\right) \mathcal{I}_{v_{i}}(s) \\
\quad+\bar{u}_{i-1}(t-s, 1) \mathcal{I}_{u_{i}}(s) \mathrm{d} s .
\end{gathered}
$$

where for all $s \in\left[0, \frac{1}{\lambda_{i}}\right]$ :

$$
\left\{\begin{aligned}
\mathcal{I}_{v_{i}}(s) & =\rho_{i, i} f_{i}\left(1-\lambda_{i} s\right)+q_{i, i} \lambda_{i} L_{i}^{z w}\left(1, \lambda_{i} s\right) \\
& +\int_{\lambda_{i} s}^{1} L_{i}^{z w}(1, \xi) f_{i}\left(\xi-\lambda_{i} s\right) \mathrm{d} \xi \\
\mathcal{I}_{u_{i}}(s) & =\rho_{i, i} h_{i}\left(1-\lambda_{i} s\right)+q_{i, i-1} \lambda_{i} L_{i}^{z w}\left(1, \lambda_{i} s\right) \\
& +\int_{\lambda_{i} s}^{1} L_{i}^{z w}(1, \xi) h_{i}\left(\xi-\lambda_{i} s\right) \mathrm{d} \xi
\end{aligned}\right.
$$

Assuming that $\bar{u}_{i-1}(t, 1)$ is known on a time interval $[t, t+$ $\left.\tau-\tau_{i}\right]$, it becomes possible to compute $\bar{d}_{i}$ on the time interval $\left[t, t+\tau-\tau_{i+1}\right]$ and $\bar{u}_{i}(t, 1)$ on $\left[t, t+\tau-\tau_{i+1}\right]$. 
More precisely, for $\tau>\tau_{i}+\frac{1}{\mu_{i}}=\tau_{i+1}$, the expressions 27)(30) define the linear operators $\mathcal{L}_{u_{i}}, \mathcal{L}_{d_{i}}$ that verify Lemma1 1 Moreover, with Assumption 2 , we can now estimate $\bar{v}_{i+1}(t, 0)$ on the time window $\left[t, t+\tau-\tau_{i+1}\right]$ (which gives us an estimation of $\left.\bar{y}_{i+1}\right)$. This concludes the proof of Lemma 1. 350

\subsection{Estimation of the ODE state}

Consider the last subsystem and estimate delayed values of the ODE state using the interaction with the PDE network. We have the following equations:

$$
\begin{aligned}
\dot{\bar{X}}(t) & =A \bar{X}(t)+B \bar{u}_{N}(t, 1), \\
\bar{d}_{N}(t) & =C \bar{X}(t) .
\end{aligned}
$$

Assume that the functions $\bar{d}_{N}$ and $\bar{u}_{N}$ are known. Using ${ }_{36}$ the matrix $L \in \mathbb{R}^{p \times 1}$ defined in Assumption 1, we can design an observer $\hat{\bar{X}}$ for the time-shifted ODE (32), if it is not stable. Such a Luenberger-observer can be written as

$$
\dot{\dot{\bar{X}}}(t)=A \hat{\bar{X}}(t)+B \bar{u}_{N}(t, 1)-L\left(\bar{d}_{N}(t)-C \hat{\bar{X}}(t)\right) .
$$

The error between the real state and the observer, defined as $\tilde{\bar{X}}=\bar{X}-\hat{\bar{X}}$, verifies

$$
\dot{\dot{\bar{X}}}(t)=(A+L C) \tilde{\bar{X}}(t) .
$$

Since $A+L C$ is Hurwitz, the error converges towards 0 , such that the designed observer $\hat{\bar{X}}$ converges towards the real delayed state $\bar{X}(t)$

Thus, after a specific convergence delay, we can estimate accurately delayed values of the state $X(t)$.

\subsection{Recursive estimation of boundary states}

Consider now the whole time-delay system (6)- $(10)$. Recall the notation $\tau_{i}=\sum_{j=1}^{i-1} \frac{1}{\mu_{j}}$. We can recursively apply Lemma 1 to obtain the following:

Theorem 1. If $\tau \geq \tau_{N+1}$, there exist causal linear operators $\mathcal{L}_{u}^{i}, \mathcal{L}_{v}^{i+1}$ such that, for all $t>\tau+\sum_{j=1}^{i} \frac{1}{\lambda_{j}}$

$$
\begin{array}{lr}
\bar{u}_{i}(\nu, 1)=\mathcal{L}_{u}^{i}(y(\cdot), V(\cdot)), & \forall i \in \llbracket 1, N \rrbracket, \\
\bar{v}_{i+1}(\nu, 0)=\mathcal{L}_{v}^{i+1}(y(\cdot), V(\cdot)), & \forall i \in \llbracket 0, N-1 \rrbracket,
\end{array}
$$

for all $\nu \in\left[t, t+\tau-\tau_{i+1}\right]$.

Moreover, there exists a causal linear operator $\mathcal{L}_{X}$ such that for all $t>\tau+\sum_{j=1}^{N} \frac{1}{\lambda_{j}}$

$$
\bar{X}(\nu)=\mathcal{L}_{X}(y(\cdot), V(\cdot)),
$$

for all $\nu \in\left[t, t+\tau-\tau_{N}\right]$.

Proof. The proof is based on an induction argument. Note that we choose $t$ large enough so that we can properly obtain equations (27) and (30). We first consider $i=1$.
Equations (27) and (30) give us the two linear operators $\mathcal{L}_{u}^{1}$, and $\mathcal{L}_{v}^{1}$ (since $\bar{y}$ and $\bar{V}$ are both known on the time interval $[t, t+\tau])$. Using the fact that $\bar{v}_{2}(t, 0)$ and $\bar{u}_{1}(t, 1)$ can be estimated on the time horizon $\left[t, t+\tau-\tau_{2}\right]$, we can apply Lemma 1. It then becomes possible to recursively define the linear operators $\mathcal{L}_{u}^{i}, \mathcal{L}_{v}^{i}$.

This theorem, shows that Property 1 is satisfied for the system under consideration. We can then define the observer states as $\hat{\bar{u}}_{i}(t, 1)=\mathcal{L}_{u}^{i}(y(),. V(\cdot))$, and $\hat{\bar{v}}_{i}(t, 0)=$ $(\cdot), V(\cdot))$, and $X(t)=\mathcal{L}_{X}(y(\cdot), V(\cdot))$. The proposed procedure actually gives exact (and not asymptotic) delayedvalues of the boundary PDE states $u_{i}(t, 1)$, and $v_{i}(t, 0)$. However, we decide to keep the terminology 'estimation' and the notation ${ }^{\hat{}}$ to emphasize the fact these values are not directly available but computed using the proposed estimation procedure. In presence of noisy and corrupted measurement, these estimations will not be exact anymore. However, the questions on the robustness of the resulting output-feedback control law are out of the scope of this paper.

\section{Boundary-states' predictor design}

In the previous section, we used our recursive dynamics interconnection framework to estimate the PDEs' boundary states' delayed values and the ODE. However, to apply the methodology developed in [25] and design an output feedback control law for our system, we need to estimate non-delayed values of these boundary states (and even predict future values to apply the tracking procedure described in 25]). In this section, we design a predictor for the boundary states $\bar{u}_{i}(t, 1)$ and $\bar{v}_{i}(t, 0)$. The predictor will give $\tau+\sum_{j=1}^{i-1} \frac{1}{\lambda_{j}}$ ahead of time values of these delayed boundary states. We will prove the following property.

\section{Property 2. Predictability}

For all $x \in[0,1]$, it is possible to obtain a $\tau+\sum_{j=1}^{i-1} \frac{1}{\lambda_{j}}+\frac{x}{\lambda_{i}}$ units of time ahead estimation of the PDE states $\bar{u}_{i}(t, x)$ and $\bar{v}_{i}(t, x)$, and $a \tau+\sum_{j=1}^{n} \frac{1}{\lambda_{j}}$ units of time ahead estimation of the ODE $\bar{X}$, using solely the measure $y(t)$ and the control law $V(t)$. These estimations asymptotically converge towards the real predictions.
For each subsystem $i \in \llbracket 1, N \rrbracket$, we apply the following strategy:

- using a backstepping transform, we remove some in-domain couplings and localize them on the left boundary of the PDEs subsystems,

- we use the method of characteristics to rewrite the boundary states as delay-equations,

- we design a predictor and show that it matches the boundary states' expected values (see [25, 37, 26]). 
Even though it may have seemed simpler to use the back-

stepping transformation (11)-(12), it is not appropriate to design the predictors. Indeed, this transformation moves the control input inside the PDE domain in the target system. This can be seen in (18) and 19) through the functions $h_{1}$ and $k_{1}$, considering that $\bar{u}_{0}(t, 1)=V(t-\tau)$. Consequently, such a neutral formulation of the system (see (27) for instance) would involve current values of the ${ }^{405}$ actuation, which makes the prediction impossible.

\subsection{Backstepping transform}

The first step towards the design of a predictor is to express the system as a neutral equation. To do so, we follow the approach proposed in 25] and consider the following backstepping transform:

$$
\begin{array}{r}
\bar{\alpha}_{i}(t, x)=\bar{u}_{i}(t, x)-\int_{x}^{1} K_{i}^{u u}(x, \xi) \bar{u}_{i}(t, \xi) \\
\quad+K_{i}^{u v}(x, \xi) \bar{v}_{i}(t, \xi) d \xi \\
\bar{\beta}_{i}(t, x)=\bar{v}_{i}(t, x)-\int_{x}^{1} K_{i}^{v u}(x, \xi) \bar{u}_{i}(t, \xi) \\
+K_{i}^{v v}(x, \xi) \bar{v}_{i}(t, \xi) d \xi
\end{array}
$$

where the kernels $K_{i}^{*}$ are continuous functions defined on the upper triangular part of the unit square $\mathcal{T}=\{(x, y) \in$ $\left.[0,1]^{2}, x \leq y\right\}$. They satisfy the following set of equations

$$
\lambda_{i} \partial_{x} K_{i}^{u u}(x, y)+\lambda_{i} \partial_{y} K_{i}^{u u}(x, y)=-\sigma_{i}^{-}(y) K_{i}^{u v}(x, y),
$$

$$
\begin{aligned}
& \lambda_{i} \partial_{x} K_{i}^{u v}(x, y)-\mu_{i} \partial_{y} K_{i}^{u v}(x, y)=-\sigma_{i}^{+}(y) K_{i}^{u u}(x, y), \\
& \mu_{i} \partial_{x} K_{i}^{v u}(x, y)-\lambda_{i} \partial_{y} K_{i}^{v u}(x, y)=\sigma_{i}^{-}(y) K_{i}^{v v}(x, y), \\
& \mu_{i} \partial_{x} K_{i}^{v v}(x, y)+\mu_{i} \partial_{y} K_{i}^{v v}(x, y)=\sigma_{i}^{+}(y) K_{i}^{v u}(x, y), \\
& K_{i}^{u v}(x, x)=-\frac{\sigma_{i}^{+}(x)}{\lambda_{i}+\mu_{i}}, K_{i}^{v u}(x, x)=\frac{\sigma_{i}^{-}(x)}{\lambda_{i}+\mu_{i}}, \\
& K_{i}^{u u}(x, 1)=\rho_{i, i} \frac{\mu_{i}}{\lambda_{i}} K_{i}^{u v}(x, 1), \quad K_{i}^{v v}(x, 1)=0 .
\end{aligned}
$$

It has been proved in [14, 35] that this set of equations admits a unique solution. Applying the transformation (36)-37) to each subsystem (6)-(7), we obtain the target system

$$
\begin{aligned}
& \partial_{t} \bar{\alpha}_{i}(t, x)+\lambda_{i} \partial_{x} \bar{\alpha}_{i}(t, x)=f_{i}^{+}(x) \bar{v}_{i+1}(t, 0) \\
& \partial_{t} \bar{\beta}_{i}(t, x)-\mu_{i} \partial_{x} \bar{\beta}_{i}(t, x)=f_{i}^{-}(x) \bar{\alpha}_{i}(t, 1),
\end{aligned}
$$

with the boundary conditions

$$
\begin{aligned}
& \bar{\alpha}_{i}(t, 0)=q_{i, i} \bar{v}_{i}(t, 0)+q_{i, i-1} \bar{u}_{i-1}(t, 1) \\
& +\int_{0}^{1} M_{i}^{\alpha \alpha}(0, \xi) \bar{\alpha}_{i}(t, \xi)+M_{i}^{\alpha \beta}(0, \xi) \bar{\beta}_{i}(t, \xi), \\
& \bar{\beta}_{i}(t, 1)=\rho_{i, i} \bar{\alpha}_{i}(t, 1)+\rho_{i, i+1} \bar{v}_{i+1}(t, 0) .
\end{aligned}
$$

where we recall that the kernels $M_{i}^{*}$ are the inverse kernels of the kernels $K_{i}^{*}$ (see [14, 35] for details). The two gain functions $f_{i}^{-}, f_{i}^{+}$are defined by $f_{i}^{-}(x)=\lambda_{i} K_{i}^{v u}(x, 1)$ and $f_{i}^{+}(x)=-\mu_{i} \rho_{i, i+1} K_{i}^{u v}(x, 1) \forall x \in[0,1]$.

The ODE system verifies

$$
\dot{\bar{X}}(t)=A \bar{X}(t)+B \bar{u}_{N}(t, 1)
$$

Unlike the backstepping transform (11)- 12 , the backstepping transform (36)-37) preserves the terms $\bar{u}_{i}(t, 1)$ and $\bar{v}_{i}(t, 0)$ inside the target system. We will now design a predictor for the states $\bar{u}_{i}(t, 1), \bar{v}_{i}(t, 0), \bar{\alpha}_{i}(t, 0)$ and $\bar{X}(t)$.

\subsection{Neutral-type formulation of the boundary states}

We apply the method of characteristics on the target system to rewrite the boundary states $\bar{u}_{i}(t, 1), \bar{v}_{i}(t, 0)$ as solutions of difference equations. We define $\delta_{i} \doteq \frac{1}{\mu_{i}}+\frac{1}{\lambda_{i}}$. We have for $i \in \llbracket 1, N \rrbracket$

$$
\begin{aligned}
\bar{u}_{i}(t, 1)= & \bar{\alpha}_{i}\left(t-\frac{1}{\lambda_{i}}, 0\right) \\
& +\int_{0}^{\frac{1}{\lambda_{i}}} f_{i}^{+}\left(1-\nu \lambda_{i}\right) \bar{v}_{i+1}(t-\nu, 0) d \nu,
\end{aligned}
$$

Following the approach given in 32, we obtain

$$
\begin{aligned}
\bar{v}_{i}(t, 0)= & \rho_{i, i} \bar{u}_{i}\left(t-\frac{1}{\mu_{i}}, 1\right)+\rho_{i, i+1} \bar{v}_{i+1}\left(t-\frac{1}{\mu_{i}}, 0\right) \\
+ & \int_{0}^{\delta_{i}} g_{i}^{1}(\nu) \bar{\alpha}_{i}(t-\nu, 0)+g_{i}^{2}(\nu) \bar{u}_{i}(t-\nu, 1) \\
& +g_{i}^{3}(\nu) \bar{v}_{i+1}(t-\nu, 0) d \nu .
\end{aligned}
$$

The functions $g_{i}^{1}, g_{i}^{2}$ and $g_{i}^{3}$ are defined by

$$
\begin{aligned}
& g_{i}^{1}(\nu)=-\mathbb{1}_{\left[0, \frac{1}{\lambda_{i}}\right]}(\nu) \lambda_{i} M_{i}^{\beta \alpha}\left(0, \lambda_{i} \nu\right), \\
& g_{i}^{2}(\nu)=\mathbb{1}_{\left[0, \frac{1}{\mu_{i}}\right]}(\nu)\left(f_{i}^{-}\left(\mu_{i} \nu\right)-\mu_{i} \rho_{i, i} M_{i}^{\beta \beta}\left(0,1-\mu_{i} \nu\right)\right. \\
& \left.-\int_{0}^{1-\mu_{i} \nu} M_{i}^{\beta \beta}(0, \xi) f_{i}^{-}\left(\xi+\mu_{i} \nu\right) d \xi\right) \\
& g_{i}^{3}(\nu)=-\mathbb{1}_{\left[0, \frac{1}{\mu_{i}}\right]}(\nu)\left(\mu_{i} \rho_{i, i+1} M_{i}^{\beta \beta}\left(0,1-\mu_{i} \nu\right)\right) \\
& -\mathbb{1}_{\left[0, \frac{1}{\lambda_{i}}\right]}(\nu)\left(\int_{\lambda_{i} \nu}^{1} M_{i}^{\beta \alpha}(0, \xi) f_{i}^{+}\left(\xi-\lambda_{i} \nu\right) d \xi\right)
\end{aligned}
$$

where $\mathbb{1}_{\Omega}$ denotes the characteristic function of the set $\Omega$. Similarly, we obtain

$$
\begin{aligned}
& \bar{\alpha}_{i}(t, 0)=q_{i, i} \bar{v}_{i}(t, 0)+q_{i, i-1} \bar{u}_{i-1}(t, 1) \\
& +\int_{0}^{\delta_{i}} k_{i}^{1}(\nu) \bar{\alpha}_{i}(t-\nu, 0)+k_{i}^{2}(\nu) \bar{u}_{i}(t-\nu, 1) d \nu \\
& +\int_{0}^{\delta_{i}} k_{i}^{3}(\nu) \bar{v}_{i+1}(t-\nu, 0) d \nu .
\end{aligned}
$$

where the functions $k_{i}^{1}, k_{i}^{2}$ and $k_{i}^{3}$ are defined by

$$
\begin{aligned}
& k_{i}^{1}(\nu)=\mathbb{1}_{\left[0, \frac{1}{\lambda_{i}}\right]}(\nu) \lambda_{i} M_{i}^{\alpha \alpha}\left(0, \lambda_{i} \nu\right), \\
& k_{i}^{2}(\nu)=\mathbb{1}_{\left[0, \frac{1}{\mu_{i}}\right]}(\nu)\left(\mu_{i} \rho_{i, i} M_{i}^{\alpha \beta}\left(0,1-\mu_{i} \nu\right)\right.
\end{aligned}
$$




$$
\begin{aligned}
& \left.+\int_{0}^{1-\mu_{i} \nu} M_{i}^{\alpha \beta}(0, \xi) f_{i}^{-}\left(\xi+\mu_{i} \nu\right) d \xi\right) \\
& k_{i}^{3}(\nu)=\mathbb{1}_{\left[0, \frac{1}{\mu_{i}}\right]}(\nu)\left(\mu_{i} \rho_{i, i+1} M_{i}^{\alpha \beta}\left(0,1-\mu_{i} \nu\right)\right) \\
& +\mathbb{1}_{\left[0, \frac{1}{\lambda_{i}}\right]}(\nu)\left(\int_{\lambda_{i} \nu}^{1} M_{i}^{\alpha \alpha}(0, \xi) f_{i}^{+}\left(\xi-\lambda_{i} \nu\right) d \xi\right) .
\end{aligned}
$$

Note that the given expressions are still valid for $i=0$ (respectively $i=N)$, using $q_{1,0} \bar{u}_{0}(t, 1)=V(t-\tau)$ (respectively $\left.\bar{v}_{N+1}=X(t)\right)$.

\subsection{Estimation of the state $\bar{\alpha}_{i}(t, 0)$}

In order to predict future values of the different boundary states $\bar{u}_{i}(t, 1), \bar{v}_{i}(t, 0), \bar{\alpha}_{i}(t, 0)$ and $\bar{X}(t)$, we need to know their initial values at time $t$. If this has already been done for the states $\bar{u}_{i}(t, 1), \bar{v}_{i}(t, 0)$, and $\bar{X}(t)$ in Section 3 (using the estimates $\hat{\bar{u}}_{i}(t, 1), \hat{\bar{v}}_{i}(t, 0)$, and $\left.\hat{\bar{X}}(t)\right)$, it is not the case for the state $\bar{\alpha}_{i}(t, 0)$. However, using equation 46 and integrating the states $\bar{\alpha}_{i}$ and $\bar{\beta}_{i}$ along the

$$
\begin{aligned}
& \bar{\alpha}_{i}(t, 0)=q_{i, i} \bar{v}_{i}(t, 0)+q_{i, i-1} \bar{u}_{i-1}(t, 1) \\
& +\int_{0}^{\frac{1}{\mu_{i}}} k_{i}^{2}(\nu) \bar{u}_{i}(t-\nu, 1) d \nu \\
& +\int_{0}^{\frac{1}{\mu_{i}}} \mu_{i} M_{i}^{\alpha \beta}\left(0,1-\mu_{i} \nu\right) \rho_{i, i+1} \bar{v}_{i+1}(t-\nu, 0) d \nu \\
& +\int_{0}^{\frac{1}{\lambda}{ }_{i}} \lambda_{i} M_{i}^{\alpha \alpha}\left(0,1-\lambda_{i} \nu\right) \bar{u}_{i}(t+\nu, 1) d \nu \\
& -\int_{0}^{\frac{1}{\lambda_{i}}}\left(\int_{0}^{1-\lambda_{i} \nu} f_{i}^{+}\left(\xi+\lambda_{i} \nu\right) M_{i}^{\alpha \alpha}(0, \xi) d \xi\right) \bar{v}_{i+1}(t+\nu, 0) d \nu .
\end{aligned}
$$

Replacing the values of the different states by their estimates and using Property 1, we can have an estimation of the functions $\bar{u}_{i}$ and $\bar{v}_{i}$ between $t$ and $t+\tau-\tau_{N}$. Thus, it is sufficient to choose $\tau$ greater than $\tau_{N}+\sup _{i}\left\{\frac{1}{\lambda_{i}}\right\}$ to

obtain an estimation of $\bar{\alpha}_{i}$. This estimator will be denoted $\hat{\bar{\alpha}}_{i}(t, 0)$.

\subsection{State prediction}

In this section, we define the state predictors $P_{\bar{v}_{i}}(t, s)$, $P_{\bar{u}_{i}}(t, s)$ and $P_{\bar{\alpha}_{i}}(t, s)$. Following the approach of [25], we define the total transport delay $\delta_{N} \doteq \sum_{i=1}^{N} \delta_{i}=\sum_{i=1}^{N} \frac{1}{\mu_{i}}+$ $\frac{1}{\lambda_{i}}$. We define for $t \geq 0$ and $s \in\left[t-\tau-\delta_{N}-\sum_{j=1}^{i-1} \frac{1}{\lambda_{j}}, t\right]$, $P_{\bar{v}_{i}}(t, s)$ (resp. $\left.P_{\bar{\alpha}_{i}}(t, s)\right)$ as the state prediction of future values of $\bar{v}_{i}(t, 0)\left(\operatorname{resp} . \bar{\alpha}_{i}(t, 0)\right)$ (ahead a time $\left.\tau+\sum_{j=1}^{i-1} \frac{1}{\lambda_{j}}\right)$, and for $s \in\left[t-\tau-\delta_{N}-\sum_{j=1}^{i} \frac{1}{\lambda_{j}}, t\right] P_{\bar{u}_{i}}(t, s)$ as the state prediction of future values of $\bar{u}_{i}(t, 1)$ (ahead a time $\tau+$

$\left.\sum_{j=1}^{i} \frac{1}{\lambda_{j}}\right)$, by the set of equations $58-60$.

$$
P_{\bar{\alpha}_{i}}(t, s)=\left\{\begin{array}{l}
\hat{\bar{\alpha}}_{i}\left(s+\tau+\sum_{j=1}^{i-1} \frac{1}{\lambda_{j}}, 0\right) \\
\quad \text { if } s \in\left[t-\delta_{N}-\tau-\sum_{j=1}^{i-1} \frac{1}{\lambda_{j}}, t-\tau-\sum_{j=1}^{i-1} \frac{1}{\lambda_{j}}\right] \\
q_{i, i} P_{\bar{v}_{i}}(t, s)+q_{i, i-1} P_{\bar{u}_{i-1}}(t, s) \\
+\int_{0}^{\delta_{i}} k_{i}^{1}(\nu) P_{\bar{\alpha}_{i}}(t, s-\nu)+k_{i}^{2}(\nu) P_{\bar{u}_{i}}\left(t, s-\nu-\frac{1}{\lambda_{i}}\right) d \nu \\
+\int_{0}^{\delta_{i}} k_{i}^{3}(\nu) P_{\bar{v}_{i+1}}\left(t, s-\nu-\frac{1}{\lambda_{i}}\right) d \nu \text { otherwise, }
\end{array}\right.
$$$$
P_{\bar{v}_{i}}(t, s)=\left\{\begin{array}{l}
\hat{\bar{v}}_{i}\left(s+\tau+\sum_{j=1}^{i-1} \frac{1}{\lambda_{j}}, 0\right) \\
\quad \text { if } s \in\left[t-\delta_{N}-\tau-\sum_{j=1}^{i-1} \frac{1}{\lambda_{j}}, t-\tau-\sum_{j=1}^{i-1} \frac{1}{\lambda_{j}}\right] \\
\rho_{i, i+1} P_{\bar{v}_{i+1}}\left(t, s-\frac{1}{\lambda_{i}}-\frac{1}{\mu_{i}}\right)+\rho_{i, i} P_{\bar{u}_{i}}\left(t, s-\frac{1}{\lambda_{i}}-\frac{1}{\mu_{i}}\right) \\
+\int_{0}^{\delta_{i}} g_{i}^{1}(\nu) P_{\bar{\alpha}_{i}}(t, s-\nu)+g_{i}^{2}(\nu) P_{\bar{u}_{i}}\left(t, s-\nu-\frac{1}{\lambda_{i}}\right) d \nu \\
+\int_{0}^{\delta_{i}} g_{i}^{3}(\nu) P_{\bar{v}_{i+1}}\left(t, s-\nu-\frac{1}{\lambda_{i}}\right) d \nu \text { otherwise, }
\end{array}\right.
$$

$$
P_{\bar{u}_{i}}(t, s)=\left\{\begin{array}{l}
\hat{\bar{u}}_{i}\left(s+\tau+\sum_{j=1}^{i} \frac{1}{\lambda_{j}}, 0\right) \\
\quad \text { if } s \in\left[t-\delta_{N}-\tau-\sum_{j=1}^{i} \frac{1}{\lambda_{j}}, t-\tau-\sum_{j=1}^{i} \frac{1}{\lambda_{j}}\right] \\
P_{\bar{\alpha}_{i}}(t, s)+\int_{0}^{\frac{1}{\lambda_{i}}} f_{i}^{+}\left(1-\nu \lambda_{i}\right) P_{\bar{v}_{i+1}}(t, s-\nu) d \nu \\
\text { otherwise, }
\end{array}\right.
$$

Note that $P_{\bar{\alpha}_{1}}(t, s)$ is well-defined and causal, using the convention $q_{1,0} P_{\bar{u}_{0}}(t, s)=V(s), s \in\left[t-\tau-\delta_{N}, t\right]$. The right-end of the chain is interconnected with the ODE, described by equation (5). We can also define $P_{\bar{X}}(t, s)$ (see [10, 37]) as the state prediction of future values of $X(t)$ (ahead a time $\left.\sum_{j=1}^{N} \frac{1}{\lambda_{j}}\right)$, for $s \in\left[t-\delta_{N}-\sum_{j=1}^{N} \frac{1}{\lambda_{j}}, t\right]$, by the set of equation 61.

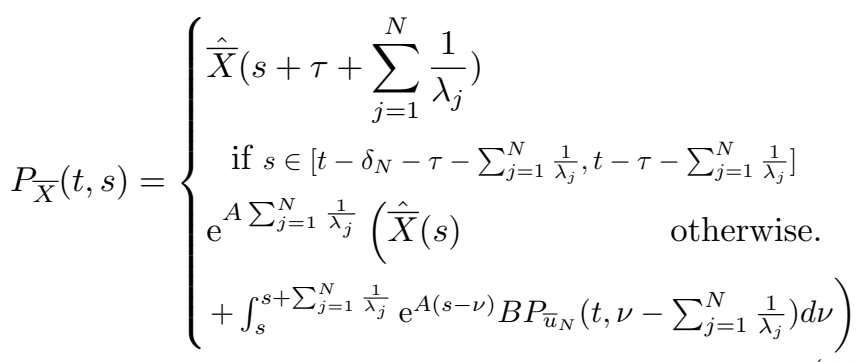

From these definitions, we immediately have:

$$
\begin{aligned}
& P_{\bar{\alpha}_{i}}(t, s)=\hat{\bar{\alpha}}_{i}\left(s+\tau+\sum_{j=1}^{i-1} \frac{1}{\lambda_{j}}, 0\right), s \in\left[t-\tau-\delta_{N}-\sum_{j=1}^{i-1} \frac{1}{\lambda_{j}}, t\right], \\
& P_{\bar{v}_{i}}(t, s)=\hat{\bar{v}}_{i}\left(s+\tau+\sum_{j=1}^{i-1} \frac{1}{\lambda_{j}}, 0\right), s \in\left[t-\tau-\delta_{N}-\sum_{j=1}^{i-1} \frac{1}{\lambda_{j}}, t\right], \\
& P_{\bar{u}_{i}}(t, s)=\hat{\bar{u}}_{i}\left(s+\tau+\sum_{j=1}^{i} \frac{1}{\lambda_{j}}, 1\right), s \in\left[t-\tau-\delta_{N}-\sum_{j=1}^{i} \frac{1}{\lambda_{j}}, t\right],
\end{aligned}
$$


$P_{\bar{X}}(t, s)=\hat{\bar{X}}\left(s+\tau+\sum_{j=1}^{N} \frac{1}{\lambda_{j}}\right), s \in\left[t-\tau-\delta_{N}-\sum_{j=1}^{N} \frac{1}{\lambda_{j}}, t\right]$.

To numerically compute the predictions, we first initialize the predictors using the estimations obtained in Section 3 .

These values are stored in a buffer. Then, it becomes possible to directly use equations (59)-(61) to compute the prediction at the next time step. Iterating gives the prediction on the required intervals. Note, that the conver- ${ }^{465}$ gence of the predictor for the ODE state is guaranteed by (30] Part 1, Chapter 3).

\subsection{Observer design}

We can now use the predictions of the PDEs bound- ${ }^{470}$ ary states and of the ODE to design a state observer for the whole system. With the predictors designed in Section 4 . we have access to the values of the real boundary states $u_{i}(t, 1)$ on the time interval $\left[t, t+\sum_{j=1}^{i} \frac{1}{\lambda_{j}}\right]$, and $v_{i}(t, 0)$ on the time interval $\left[t, t+\sum_{j=1}^{i-1} \frac{1}{\lambda_{j}}\right]$.

We then use the predictors $P_{\bar{u}_{i}}(t, s), P_{\bar{v}_{i}}(t, s)$ in the delayed equations

$$
\begin{aligned}
\bar{\alpha}_{i}(t, x) & =\bar{\alpha}_{i}\left(t-\frac{x}{\lambda_{i}}, 0\right)+\int_{0}^{\frac{x}{\lambda_{i}}} f_{i}^{+}\left(x-\lambda_{i} \nu\right) \bar{v}_{i+1}(t-\nu, 0) d \nu_{480} \\
\bar{\beta}_{i}(t, x) & =\rho_{i, i} \bar{u}_{i}\left(t-\frac{1-x}{\mu_{i}}, 1\right)+\rho_{i, i+1} \bar{v}_{i+1}\left(t-\frac{1-x}{\mu_{i}}, 0\right) \\
+ & \int_{0}^{\frac{1-x}{\mu_{i}}} f_{i}^{-}\left(x+\mu_{i} \nu\right) \bar{u}_{i}(t-\nu, 1) d \nu
\end{aligned}
$$

to predict future values of the states $\bar{\alpha}_{i}(t, x)$ and $\bar{\beta}_{i}(t, x)$. Finally, using the invertibility of the backstepping transformation (36)-(37), we can compute future values of the states $\bar{u}_{i}(t, x), \bar{v}_{i}(t, x)$, for all $x \in[0,1]$, and thus finally have access to the whole state $u_{i}, v_{i}$. More specifically, we have proved the following theorem.

Theorem 2. Consider the system (1)-(5) $(i \in \llbracket 1, N \rrbracket)$, and assume that the measure $y(s)=v_{1}(s, 0)$ and the control law $V(s)$ are known on a time interval $s \in[0, t], t>0$. Then, there exist predictor functions $P_{\bar{v}_{i}}, P_{\bar{u}_{i}}$ and $P_{\bar{X}}$ such that for all $x \in[0,1], P_{\bar{v}_{i}}(t, x)=\bar{v}_{i}\left(t+\tau+\sum_{j=1}^{i-1} \frac{1}{\lambda_{j}}+\frac{x}{\lambda_{i}}, x\right)$, $P_{\bar{u}_{i}}(t, x)=\bar{u}_{i}\left(t+\tau+\sum_{j=1}^{i-1} \frac{1}{\lambda_{j}}+\frac{x}{\lambda_{i}}, x\right), P_{\bar{X}}(t)=\bar{X}(t+\tau+$ $\left.\sum_{j=1}^{N} \frac{1}{\lambda_{j}}\right)$.

Such predictors satisfy Property 2 .

\section{Output-feedback control law design}

In this section, we design an output-feedback control law that exponentially stabilizes the system. We follow the recursive dynamics interconnection framework proposed in [25]. The strategy is the following. Using the previouslydesigned predictors, we have access to the values of $X(t)$. To stabilize the ODE, we want to make $u_{N}(t, 1)$ converge towards $\Phi_{N}(t)=K X(t)$ (where $K$ is defined in Assumption 3). If we have trackability of subsystem $N$, in the presence of perturbation (that will converge towards 0 ), we can then compute the virtual command $\hat{V}_{N-1}(t)$, towards which $u_{N-1}(t, 1)$ must converge. We then go up the whole chain until the first subsystem to recursively design the output feedback control law $V(t)$.

\subsection{State feedback controller}

This section will recall the results on stabilization and trackability from [25. We will then use them to design the output feedback control law.

Let us define $\hat{V}_{i}(t)=q_{i, i-1} u_{i-1}(t, 1)$ as the virtual input acting on subsystem $i \in \llbracket 1, N \rrbracket$, and $\Phi_{i}(t)$ an arbitrary virtual output of subsystem $i$. Let us also denote $\chi_{i}(t)$ the action of the $(i+1)^{t h}$ subsystem on the $i^{\text {th }}$ subsystem. This function will be called virtual disturbance acting on the $i^{t h}$ subsystem. We have $\chi_{i}(t)=\rho_{i, i+1} v_{i+1}(t, 0)$ We have the two following properties:

\section{Property 3. Stabilizability :}

For all $i \leq N$, in the absence of the virtual disturbance $\chi_{i}\left(\right.$ i.e. $\left.\chi_{i}(t) \equiv 0\right)$, the $i^{\text {th }}$ subsystem subject to the virtual actuation $\hat{V}_{i}$ must be stabilizable by a state-feedback law. More precisely, there exists an operator $\mathcal{K}_{i}$, such that: $\hat{V}_{i}(t)=\mathcal{K}_{i}\left(u_{i}(t, \cdot), v_{i}(t, \cdot)\right) \Longrightarrow\left\|u_{i}(t), v_{i}(t)\right\|_{L^{2}} \underset{t \rightarrow+\infty}{\longrightarrow}$ 0. Moreover, there exists an operator $\mathcal{K}_{N+1}$, such that : $\hat{V}_{N+1}(t)=\mathcal{K}_{N+1}(X(t)) \Longrightarrow\|X\| \underset{t \rightarrow+\infty}{\longrightarrow} 0$.

\section{Property 4. Trackability :}

Consider a subsystem $i \leq N$ and define $\Phi_{i} \in L_{\mathrm{loc}}^{2}\left(\mathbb{R}^{+}\right)$an arbitrary known function. Let us assume that the virtual disturbance $\chi_{i}$ acting on this subsystem is known. Then, there exists a control law $\hat{V}_{i}$ that exponentially tracks the function $\hat{V}_{i+1}(t)$ to the desired function $\Phi_{i}(t)$. Moreover, if $\chi_{i}(t) \equiv \Phi_{i}(t) \equiv 0$, then, such a control law stabilizes the $i^{\text {th }}$ subsystem. More precisely, there exists an operator $\mathcal{L}_{i}$, such that :

$$
\begin{array}{r}
\hat{V}_{i}(t)=\mathcal{K}_{i}\left(u_{i}(t, \cdot), v_{i}(t, \cdot)\right)+\mathcal{L}_{i}\left(\Phi_{i}\left(t+\frac{1}{\lambda_{i}}\right), \chi_{i}\left(\left[t, t+\frac{1}{\lambda_{i}}\right]\right)\right) \\
\Longrightarrow \bar{u}_{i}(t, 1) \underset{t \rightarrow+\infty}{\longrightarrow} \Phi_{i}(t)
\end{array}
$$

where $\mathcal{K}_{i}$ is the operator defined by Property 3.

Notice that the operator $\mathcal{L}_{i}$ may require future values of the states. This justifies the design of predictors. Following [25], we can define the linear operators $\mathcal{K}_{i}, \mathcal{L}_{i}$, pour $i \leq$ $N$ as

$\mathcal{K}_{i}\left\{\begin{aligned} &\left(L^{2}([0,1], \mathbb{R})\right)^{2} \longrightarrow \mathbb{R} \\ &(f, g) \longmapsto-q_{i, i} f(., 0) \\ &+\int_{0}^{1} K_{i}^{u u}(0, y) f(., y)+K_{i}^{u v}(0, y) g(., y) \mathrm{d} y,\end{aligned}\right.$

$\mathcal{L}_{i}\left\{\begin{array}{l}\left(L^{2}\left(\mathbb{R}^{+}, \mathbb{R}\right)\right)^{2} \longrightarrow \mathbb{R} \\ (f, g) \longmapsto \frac{1}{q_{i, i+1}} f\left(.+\frac{1}{\lambda_{i}}\right) \\ \quad+\mu_{i} \rho_{i, i+1} \int_{0}^{\frac{1}{\lambda_{i}}} K_{i}^{u v}\left(x-\lambda_{i} s, 1\right) g\left(.+\frac{1}{\lambda_{i}}-s, 0\right) \mathrm{d} s,\end{array}\right.$ 
where the kernels $K_{i} \cdot$ are defined by equations (38)-43). Finally, the operator $\mathcal{K}_{N+1}(X)$ is simply defined by $\mathcal{K}_{N+1}(X)=K X$ (where the matrix $K$ is defined in Assumption 3). Under Assumption 3 and Assumption 4, 25] proved that the operators $\mathcal{K}_{i}$, and $\mathcal{L}_{i}$ verify Property $3{ }_{95}$ and Property 4 . Indeed, if we consider the backstepping transformation

$$
\begin{aligned}
& \left(\begin{array}{c}
\alpha_{i}(t, x) \\
\beta_{i}(t, x)
\end{array}\right)=\left(\begin{array}{l}
u_{i}(t, x) \\
v_{i}(t, x)
\end{array}\right) \\
& -\int_{x}^{1}\left(\begin{array}{ll}
K_{i}^{u u}(x, y) & K_{i}^{u v}(x, y) \\
K_{i}^{v u}(x, y) & K_{i}^{v v}(x, y)
\end{array}\right)\left(\begin{array}{l}
u_{i}(t, y) \\
v_{i}(t, y)
\end{array}\right) d y
\end{aligned}
$$

and choose $\hat{V}_{i}(t)=V_{i}^{t r}(t)+\hat{V}_{i}^{B S}(t)$, where $V_{i}^{t r}(t)=\mathcal{L}_{i}\left(\Phi_{i}(t+\right.$ $\left.\left.\frac{1}{\lambda_{1}}\right), \chi_{i}\left(\left[t, t+\frac{1}{\lambda_{i}}\right]\right)\right), \hat{V}_{i}^{B S}=\mathcal{K}_{i}\left(u_{i}(t, \cdot), v_{i}(t, \cdot)\right)$, we can map the system (1) $-(5)$ to the target system

$$
\begin{aligned}
& \partial_{t} \alpha_{i}+\lambda_{i} \partial_{x} \alpha_{i}=-\mu_{i} K_{i}^{u v}(x, 1) \chi_{i}(t) \\
& \partial_{t} \beta_{i}-\mu_{i} \partial_{x} \beta_{i}=\lambda_{i} K_{i}^{v u}(x, 1) \alpha_{i}(t, 1),
\end{aligned}
$$

with the boundary conditions

$$
\begin{aligned}
\alpha_{i}(t, 0) & =V_{i}^{t r}(t), \\
\beta_{i}(t, 1) & =\rho_{i, i} \alpha_{i}(t, 1)+\chi_{i}(t) .
\end{aligned}
$$

Applying the method of characteristics on equation (64), we directly have $\alpha_{i}(t, 1)=\Phi_{i}(t)$. If the functions $\Phi_{i}$ and $\chi_{i}$ converge to zero, so does the state $\alpha_{i}(t, 1)$ and so does515 the whole $i^{t h}$-subsystem due to its transport structure. In addition the following theorem has been proved in 25

Theorem 3. Existence of a state-feedback controller Consider the system (1)-(5) and let us assume that the properties 2, 3, and 4, are verified. Then, there exists $a_{520}$ state-feedback control law $V_{\Xi}^{s}(t)$ that exponentially stabilizes the system (1)-(5) in the sense of the $\Xi$-norm.

The control law defined in 25] uses state-measurement based predictors. Here, we choose to modify such a control law using the observers and predictors designed in the previous section. More precisely, let us recursively define the sequence $\hat{V}_{i}$ by

$$
\hat{V}_{N+1}(t)=K P_{\bar{X}}\left(t+\tau-\sum_{j=1}^{N} \frac{1}{\lambda_{j}}\right),
$$

and for $i \in \llbracket 1, N \rrbracket$

$$
\begin{gathered}
\hat{V}_{i}(t)=\mathcal{K}_{i}\left[P_{\bar{u}_{i}}\left(t+\tau-\sum_{j=1}^{i-1} \frac{1}{\lambda_{j}}-\frac{x}{\lambda_{i}}, x\right),\right. \\
\left.P_{\bar{v}_{i}}\left(t+\tau-\sum_{j=1}^{i-1} \frac{1}{\lambda_{j}}-\frac{x}{\lambda_{i}}, x\right)\right] \\
+\mathcal{L}_{i}\left(\hat{V}_{i+1}\left(t+\frac{1}{\lambda_{i}}\right), P_{\bar{v}_{i+1}}\left(t+\tau-\sum_{j=1}^{i-1} \frac{1}{\lambda_{j}}, 0\right)\right),
\end{gathered}
$$

where the operators $\mathcal{K}_{i}$ and $\mathcal{L}_{i}$ are defined by equation 62 and the predictors $P_{\bar{u}_{i}}, P_{\bar{v}_{i}}$ and $P_{\bar{X}}$ are defined in Theorem 2. We finally define the control law $V_{\Xi}(t)$ as

$$
V_{\Xi}(t)=\hat{V}_{1}(t)
$$

In the next section, we show that such an output-feedback control law stabilizes the system (1)-(5).

\subsection{Output-feedback control law}

The output-feedback law 70 is similar to the statefeedback law designed in 25, except that we now define the different predictors using the available measurements. To combine the existing stabilizing feedback-law with the proposed predictor-observers, we need additional properties. Let us denote $V_{\Xi}^{s}(t)$, the state-feedback control law designed in 25. This state-feedback control law would correspond to 70 except that the measurement-based predictors are replaced by state-predictors as designed in 25].

Property 5. If the control input $V_{\Xi}(t)$ asymptotically converges towards $V_{\Xi}^{s}(t)$, then it stabilizes the system (1)-(5) in the sense of the $\Xi$-norm.

This property holds for the system under consideration. This can be seen by expressing the whole system in its neutral form 32 and defining the difference between $V_{\Xi}(t)$ and $V_{\Xi}^{s}(t)$ as a disturbance. Then, using the variation-ofconstants formula for a neutral differential equation (see 33. page 31 ), we can guarantee the stabilization of the system (1)-(5). More details for complete proof can be found in 38 . We are now able to prove that the outputfeedback control law $V_{\Xi}(t)$ stabilizes the system (1)-(5).

Theorem 4. Consider the system (1)-(5). If Properties 1. 2, 3, 4 and 5 are verified, then the output-feedback control law $V_{\Xi}(t)=\hat{V}_{1}(t)$ defined by 70 exponentially stabilizes the system (1)-(5) in the sense of the $\Xi$-norm.

Proof. The control law $V_{\Xi}(t)$ is well-defined and causal due to the definition of the different predictors (Property 2.) Let us consider that the predictors provide exact values of the PDEs and ODE states' future values. The corresponding control law is $V_{\Xi}^{s}(t)$. Then, applying Property 4 on each subsystem, we obtain that $u_{N}(t, 1)$ exponentially converges to $\mathcal{K}_{N+1}(X(t))$. Consequently, $X(t)$ exponentially converges to zero. Using Property 4, we can recursively show that each subsystem exponentially converges to zero starting from $i=(n-1)$. Thus, the control law $V_{\Xi}(t)$ designed with exact predictions would stabilize the system (1)-(5). We now need to show that such a result still holds when using output measurements in the predictors' definition. Using Property 2, the designed predictors asymptotically converge towards the states' real future values. It is finally sufficient to apply Property 5 to conclude the proof. 
Note that Properties 1 and 3 are not directly used in the proof since they are usually require to show Properties 2 and 4. The formulation of Theorem 4 is extremely generic and does enforce any restriction on the control design. We

545 have proposed throughout the paper a backstepping-based controller but any other alternative design could be proposed as long as Properties 1, 2, 3, 4 and 5 hold. Most of these properties correspond to observability/predictability ${ }^{600}$ and controllability/trackability properties for each subsystem. Thus, the proposed recursive dynamics framework can be easily adapted for different interconnections, including non-scalar PDEs subsystems or ODEs inside the chain (and not just at the boundaries). Such generalizabil- ${ }^{605}$ ity is one of the main assets of the proposed framework.

Remark 2. The proposed control design completely neglects the robustness aspects. As shown in [27], the operators $\mathcal{K}_{i}$ defined in $(62)$, by cancelling the reflection terms, ${ }_{610}$ may lead to zero (delay-)robustness margins. Such a control law would consequently not be suitable for implementation on real systems. This lack of delay-robustness margins is related to the fact that we have a non-strictly-proper control operator. Different approaches can be considered to $0^{615}$ tackle this limitation. In [27], the authors suggested canceling only a part of the boundary reflection. However, this implies that the design should be completely modified since the trackability property (Property 4) would become extremely difficult to prove. More recently, an alternative ap-620 proach was suggested in [39]. The current output-feedback law could be combined with a well-tuned low-pass filter it strictly proper (and consequently delay-robust). The design of such a low-pass filter is made possible due to Assumption 5, which guarantees that the open-loop sys-625 tem is naturally exponentially stable at high frequencies. One of the advantages of using a low-pass filter is to allow good performance at low frequencies while guaranteeing robustness margins at high frequencies. In that sense, it offers additional degrees of freedom to enable trade-offs ${ }^{630}$ between performance and robustness. However, we believe that such a robustness analysis is out of the scope of this paper and will be the purpose of future research.

\section{Applications}

\subsection{Simulation results}

In this section, we illustrate the efficiency of the recursive dynamics interconnection framework presented in this article through simulations. We implemented the strategy described herein using Matlab. The simulations were made for two PDE subsystems $N=2$ coupled with a scalar ODE system. They were simulated on a 40 s time scale, with 51 spatial discretization points (and a CFL number equal to 590 1).

First, the coefficients are chosen such that the different subsystems are (slightly) unstable in open-loop. Their numerical values are $\left(\begin{array}{l}\lambda_{1} \\ \lambda_{2}\end{array}\right)=\left(\begin{array}{l}1 \\ 2\end{array}\right),\left(\begin{array}{l}\mu_{1} \\ \mu_{2}\end{array}\right)=\left(\begin{array}{l}1.3 \\ 1.8\end{array}\right),\left(\begin{array}{c}\sigma_{1}^{+} \\ \sigma_{2}^{-}\end{array}\right)=$ $\left(\begin{array}{c}1 \\ -0.3\end{array}\right),\left(\begin{array}{l}\sigma_{1}^{-} \\ \sigma_{2}^{-}\end{array}\right)=\left(\begin{array}{l}0.4 \\ 0.7\end{array}\right),\left(\begin{array}{ll}q_{11} & q_{12} \\ q_{21} & q_{22}\end{array}\right)=\left(\begin{array}{cc}0.5 & 0 \\ 0.3 & 0.7\end{array}\right),\left(\begin{array}{ll}\rho_{11} & \rho_{12} \\ \rho_{21} & \rho_{22}\end{array}\right)=$ $\left(\begin{array}{cc}0.275 & 0.4 \\ 0 & 0.4\end{array}\right)$. The ODE system is defined by $A=0.1, B=$ $0.1, C=0.1$. The different assumptions (Assumptions 1$5)$ are satisfied, and we chose the matrices $K$ and $L$ as $K_{\text {Hurwitz }}=-3, L_{\text {Hurwitz }}=-5$. Beforehand, the direct kernel gains $L_{i}$ corresponding to the invertible Volterra transform (11)-12 are computed using the successive approximation technique 40. Their values are stored in matrices whose dimension is directly defined by the number of discretization points (here 51). The computation of these kernels require 22 iterations to converge $\left(\epsilon=10^{-8}\right)$ and about $35 \mathrm{~s}$. Then, we compute the functions $f_{i}, g_{i}, h_{i}, k_{i}$ are computed. The integral terms are obtained with a trapezoidal method. Similar techniques are used to obtain the remaining kernels and the coupling terms $g_{i}^{j}, k_{i}^{j}, f_{i}^{+}, f_{i}^{-}$. All these function values are computed off-lined and do not need to be updated while running the closed-loop simulations.

Next, we can simulate the evolution of the system. Note that the whole interconnected system remains unstable in open-loop. The initial conditions of the states are constant functions $u_{i}(0,)=.v_{i}(0,)=$.0.2 and $X_{0}=1$. The observer values are initialised to 0 . The classical finite volume method based on a Godunov scheme 41 has been used to simulate all PDE systems' evolution. The ODE state is updated at each iteration using the Matlab medium order method ode45. After a delay $\tau_{i}+\sum \frac{1}{\lambda_{i}}$, the values of the delayed boundary states are computed. After a delay $\tau>\tau_{N}+\sup _{i}\left\{\frac{1}{\lambda_{i}}\right\}$, at each iteration, a buffer containing the values of the predictor is updated with the boundary state estimations, and new values of the predictor are computed. The value of the control law is computed accordingly.

We have pictured in Figure 4 the evolution of the $\Xi$-norm defined in section 2.2. As expected, the control law efficiently stabilizes the system. We have also pictured in Figure 5, the time evolution of the control effort. One can notice that we only act on the system once we are able to compute the predicted values of the different parameters. This explains the observed offset. The effect of saturation is also visible. Finally, we have plotted the time-evolution of the state $u_{2}(t, x)$ in Figure 6 .

\subsection{Illustrative example: axial motion of a drilling system}

In this section, we show how our recursive dynamics framework could be applied to an industrial problem. More precisely, we consider a (simplified) model that describes the axial motion of a drilling device. In this application case, we only focus on the estimation of the state using the proposed recursive estimation framework as described in Section 3 Indeed, our objective is not to propose a complete test case studies but to give some insights on how our methodology could be applied to industrial applications. We only considered the estimation problem here as it is known to be one of the most important problems in drilling [42. We consider the ideal 


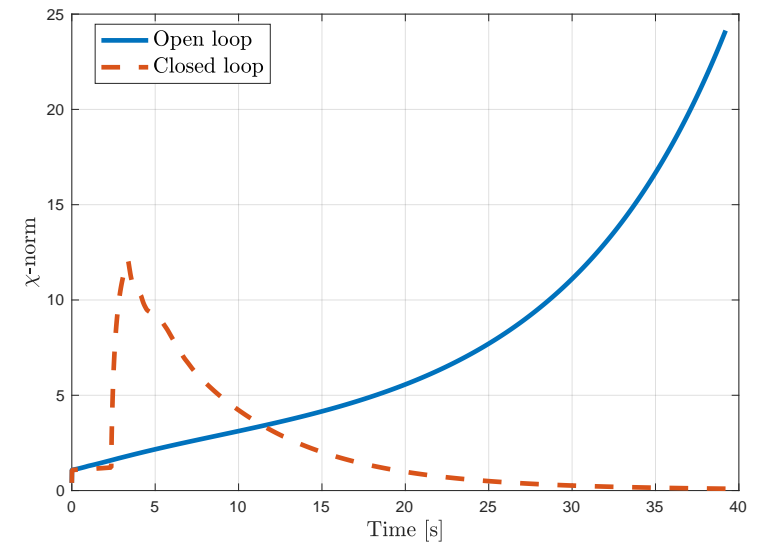

Figure 4: Evolution of the $\Xi$-norm of the open-loop system (1)(5) and of the closed-loop system using the control law defined in Theorem 4

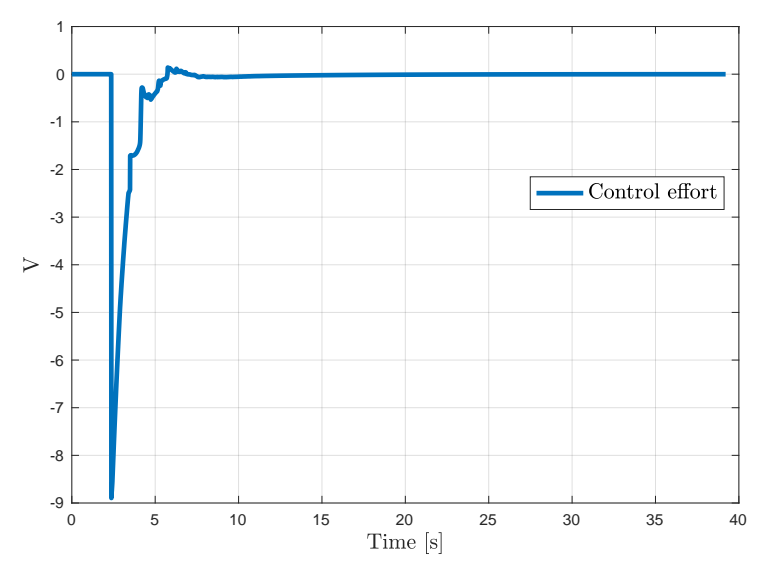

Figure 5: Time evolution of the (saturated) control effort

case of a vertical well and neglect the Coulomb friction. A mathematical model to describe the drill-string axial dynamics can be found in [42, 43]. The drilling system we consider is composed of three parts: a top drive, the drill-string and the drill-bit. The drill-string may have a bottom hole assembly (BHA) consisting of a heavier pipe at the bottom. Let us denote $\xi(t, x)$ the axial displacement of the drill-string. It is a function of $(t, x)$ evolving in $\{(t, x) \mid 0<t<T, x \in[0, L]\}$ (where $T$ is a positive time). The axial force associated to $\xi$ can be found from the strain, given as the local relative compression:

$$
w(t, x)=A E \frac{(\xi(t, x)-\xi(t, x+d x))}{d x},
$$

$A$ being the cross-sectional area of the drill-string, $E$ being its Young's modulus and $d x \rightarrow 0$ the infinitesimal axial position increment. The axial velocity satisfies

$$
v(t, x)=\frac{\partial \xi(t, x)}{\partial t} .
$$

The axial motion satisfies the following wave PDE

$$
\frac{\partial^{2} \xi}{\partial t^{2}}(t, x)-c_{\xi}^{2} \frac{\partial^{2} \xi}{\partial x^{2}}(t, x)=-k_{\xi} \frac{\partial \xi}{\partial t}(t, x),
$$

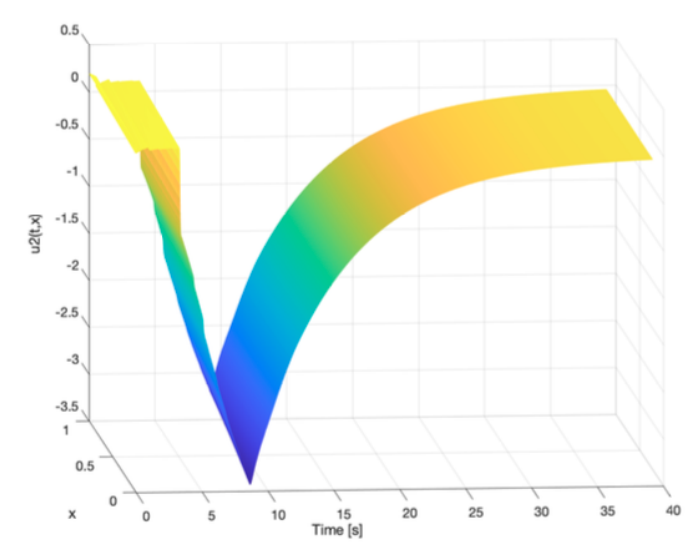

Figure 6: Time evolution of the state $u_{2}(t, x)$

where $c_{\xi}=\sqrt{\frac{E}{\rho}}, \rho$ is the pipe mass density, $E$ its Young's modulus and $k_{\xi}$ is a damping coefficient representing the viscous shear stresses acting on the pipe. The lower part of the drill-string is usually made up of heavier pipes (drill collars) that have different lengths, density, inertia or Young's modulus. This change of the characteristic line impedance may cause reflections in the traveling waves that may have a great impact on the global dynamics [4]. Let us assume we have $N$ different sections $(N \in \mathbb{N})$, and let us denote $x_{i}$ the spatial coordinate of the junction point between the $(i-1)^{\text {th }}$-section and the $i^{\text {th }}$-section. Let us denote $x_{1}=0$, $x_{N+1}=1$ and $\left(w^{i}(t, x), v^{i}(t, x)\right)$ the force and velocity along the $i^{\text {th }}$ section of the drill-string. The corresponding physical parameters will also be expressed using the superscript $^{i}$. We have the following continuity constraints at the transitions

$$
v^{i}\left(t, x_{i+1}\right)=v^{i+1}\left(t, x_{i+1}\right), \quad w^{i}\left(t, x_{i+1}\right)=w^{i+1}\left(t, x_{i+1}\right) .
$$

Let us define the Riemann invariants as

$$
\begin{gathered}
u^{i}(t, x)=\left(\frac{\partial}{\partial t} \xi^{i}(t, x)-c_{\xi}^{i} \frac{\partial}{\partial x} \xi^{i}(t, x)\right) \mathrm{e}^{\frac{k_{\xi}^{i}}{2 c_{\xi}^{i}} x}, \\
z^{i}(t, x)=\left(\frac{\partial}{\partial t} \xi^{i}(t, x)+c_{\xi}^{i} \frac{\partial}{\partial x} \xi^{i}(t, x)\right) \mathrm{e}^{-\frac{k_{\xi}^{i}}{2 c_{\xi}^{i}} x} .
\end{gathered}
$$

Consequently, on each section, equation 72 rewrites

$$
\begin{gathered}
\frac{\partial}{\partial t} u^{i}(t, x)+c_{\xi}^{i} \frac{\partial}{\partial x} u^{i}(t, x)=-\frac{k_{\xi}^{i}}{2} \mathrm{e}^{\frac{k_{\xi}^{i}}{c_{\xi}^{i}} x} z^{i}(t, x) \\
\frac{\partial}{\partial t} z^{i}(t, x)-c_{\xi}^{i} \frac{\partial}{\partial x} z^{i}(t, x)=-\frac{k_{\xi}^{i}}{2} \mathrm{e}^{-\frac{k_{\xi}^{i}}{c_{\xi}^{i}} x} u^{i}(t, x) .
\end{gathered}
$$

In the Riemann coordinates, the boundary conditions at the junctions rewrite for $i \leq N$

$$
\begin{aligned}
z^{i}\left(t, x_{i+1}\right) & =a_{1}^{i} u^{i}\left(t, x_{i+1}\right)+a_{2}^{i} z^{i+1}\left(t, x_{i+1}\right), \\
u^{i+1}\left(t, x_{i+1}\right) & =a_{3}^{i} u^{i}\left(t, x_{i+1}\right)+a_{4}^{i} z^{i+1}\left(t, x_{i+1}\right),
\end{aligned}
$$


where

$$
\begin{aligned}
& a_{1}^{i}=\frac{1-Z^{i}}{1+Z^{i}} \mathrm{e}^{-\frac{k_{\xi}^{i}}{c_{\xi}^{i}} x_{i+1}}, a_{2}^{i}=\frac{2 Z^{i}}{1+Z^{i}} \mathrm{e}^{\left(\frac{k_{\xi}^{i+1}}{2 c_{\xi}^{i+1}}-\frac{k_{\xi}^{i}}{2 c_{\xi}^{i}}\right) x_{i+1}}, \\
& a_{3}^{i}=\frac{1}{Z^{i}} a_{2}^{i}, \quad a_{4}^{i}=\frac{Z^{i}-1}{1+Z^{i}} \mathrm{e}^{\frac{k_{\xi}^{i+1}}{c_{\xi}^{i+1}} x_{i+1}},
\end{aligned}
$$

where the relative magnitude of the impedance is denoted $Z^{i}=\frac{c_{\xi}^{i}}{E^{i} A^{i}} / \frac{c_{\xi}^{i+1}}{E^{i+1} A^{i+1}}$. According to [45], we can consider that the operator controls the weight on the drill-string, such that $w_{0}(t)=w(t, 0)$. This yields

$$
u^{1}(t, 0)=z^{1}(t, 0)+\frac{2 c_{\xi}^{1}}{E^{1} A^{1}} w_{0}(t)
$$

The effect of the BHA can be lumped into an ODE coupled with the drill-string [46, 47]. Thus, the downhole boundary condition at $x=1$ can be obtained from a force balance on the lumped BHA. This yields the following downhole boundary condition

$$
\begin{aligned}
z(t, L)= & -\mathrm{e}^{-\frac{k_{\xi}}{c_{\xi}} L} u(t, L)+2 \mathrm{e}^{-\frac{k_{\xi}}{2 c_{\xi}} L} X(t), \\
\dot{X}(t)= & -\frac{a \zeta \epsilon}{M_{b} \omega_{\mathrm{bit}}} X(t)-\frac{w_{f}}{M_{b}} \\
& -\frac{E A_{s}}{2 c_{\xi} M_{b}}\left(z(t, L) \mathrm{e}^{\frac{k_{\xi}}{2 c_{\xi}} L}-u(t, L) \mathrm{e}^{-\frac{k_{\xi}}{2 c_{\xi}} L}\right),
\end{aligned}
$$

where $M_{b}$ is the mass of the lumped BHA, $\omega_{b i t}$ is the angular velocity of the bit (constant here), $w_{f}$ is the friction675 weight, $a$ is the bit radius, $\zeta$ is a characterization of the cutting angle and $\epsilon$ is the intrinsic specific energy of the rock. See [42, 43, 3] for more details on the derivation of the model. In what follows, we use the numerical values given in [3]. In the Riemann coordinates, the axial dynamics of the drilling system correspond to system (1)-(5). Consequently, it becomes possible to apply our recursive dynamics framework. We have pictured in Figure 7 the normalized error of the estimation of the ODE state $X$. As expected, once we can correctly estimate the different PDE states using our recursive dynamics framework, we ${ }^{685}$ can obtain a reliable estimation of the ODE state. However, one must be aware that such an observer requires known values of the different constant parameters. Some of them (particularly the parameters that depend on the ${ }^{690}$ nature of the drilled rock as $\epsilon$ ) may be difficult to estimate. Thus, the proposed observer should be combined with alternative techniques to estimate such constant and unknown parameters.

\section{Concluding remarks}

In this paper, we designed an output feedback control law that stabilizes a network of interconnected scalar linear hyperbolic systems coupled with an ODE at the end of the chain. We used backstepping transforms and a recur- ${ }_{705}$ sive dynamics interconnection framework to estimate the

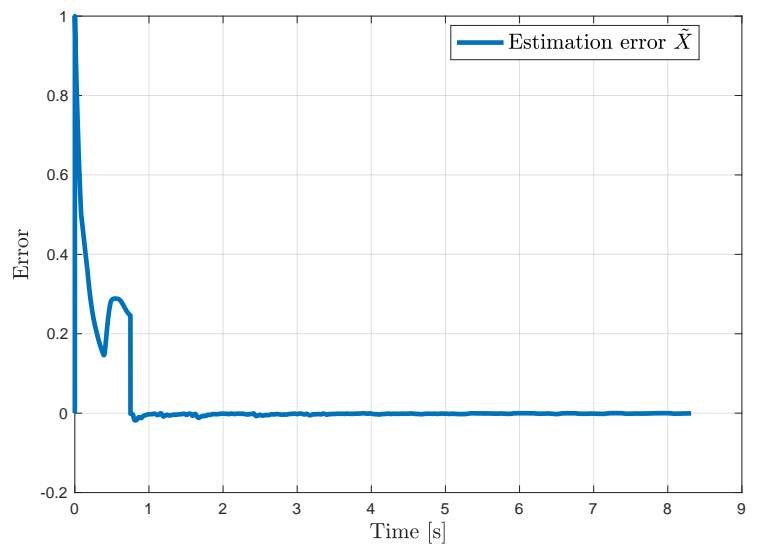

Figure 7: Normalized estimation error $\tilde{X}$ of the ODE state for the considered drilling application.

boundary states of each subsystem. We used these estimations to build predictors that allow building an output feedback control law recursively. We believe that the proposed approach could be easily adapted to different types of interconnected systems (including different subsystems or different types of chains) as long as some fundamental properties (trackability, predictability) are still verified. In future works, we will consider more general classes of networks. We want to consider non-scalar PDE subsystems and the case where ODE subsystems can be located inside the chain and specifically sandwiched between two PDE subsystems. More complex graph structures will also be the object of future research.

\section{References}

[1] G. A. de Andrade, R. Vazquez, D. J. Pagano, Backstepping stabilization of a linearized ODE-PDE rijke tube model, Automatica 96 (2018) 98-109.

[2] H. Yu, J. Auriol, M. Krstic, Simultaneous stabilization of traffic flow on two connected roads, IEEE American Control Conference (2020).

[3] J. Auriol, N. Kazemi, K. Innanen, R. Shor, Combining formation seismic velocities while drilling and a pde-ode observer to improve the drill-string dynamics estimation, in: 2020 American Control Conference (ACC), IEEE, 2020, pp. 3120-3125.

[4] D. Bresch-Pietri, M. Krstic, Adaptive output feedback for oil drilling stick-slip instability modeled by wave PDE with antidamped dynamic boundary, in: American Control Conference (ACC), 2014, IEEE, 2014, pp. 386-391.

[5] G. Bastin, J.-M. Coron, S. O. Tamasoiu, Stability of linear density-flow hyperbolic systems under PI boundary control, Automatica 53 (2015) 37-42.

[6] F. Woittennek, J. Rudolph, T. Knüppel, Flatness based trajectory planning for a semi-linear hyperbolic system of first order pde modeling a tubular reactor, PAMM 9 (1) (2009) 3-6.

[7] F. Woittennek, Flatness based feedback design for hyperbolic distributed parameter systems with spatially varying coefficients, IFAC Proceedings Volumes 46 (26) (2013) 37-42.

[8] T. Meurer, M. Zeitz, Flatness-based feedback control of diffusion-convection-reaction systems via k-summable power series, IFAC Proceedings Volumes 37 (13) (2004) 177-182.

[9] T. Strecker, O. M. Aamo, Output feedback boundary control of series interconnections of $2 \times 2$ semilinear hyperbolic systems, IFAC-PapersOnLine 50 (1) (2017) 663-670. 
[10] N. Bekiaris-Liberis, Simultaneous compensation of input and state delays for nonlinear systems, Systems and Control Letters 73 (2014) 96-102.

[11] M. Krstic, A. Smyshlyaev, Backstepping boundary control for first-order hyperbolic PDEs and application to systems with actuator and sensor delays, Systems \& Control Letters 57 (9) (2008) 750-758

[12] L. Su, W. Guo, J.-M. Wang, M. Krstic, Boundary stabilization785 of wave equation with velocity recirculation, IEEE Transactions on Automatic Control 62 (9) (2017) 4760-4767.

[13] R. Vazquez, M. Krstic, A closed-form feedback controller for stabilization of the linearized 2-d navier-stokes poiseuille system, IEEE transactions on automatic control 52 (12) (2007)790 $2298-2312$.

[14] J.-M. Coron, R. Vazquez, M. Krstic, G. Bastin, Local exponential $H^{2}$ stabilization of a $2 \times 2$ quasilinear hyperbolic system using backstepping, SIAM Journal on Control and Optimization 51 (3) (2013) 2005-2035.

725 [15] F. Di Meglio, R. Vazquez, M. Krstic, Stabilization of a system of $n+1$ coupled first-order hyperbolic linear pdes with a single boundary input, IEEE Transactions on Automatic Control 58 (12) (2013) 3097-3111.

[16] J. Auriol, Output feedback stabilization of an underactuated 800 cascade network of interconnected linear pde systems using a backstepping approach, Automatica 117 (2020) 108964.

17] F. Di Meglio, P.-O. Lamare, U. J. F. Aarsnes, Robust output feedback stabilization of an ode-pde-ode interconnection, Automatica (Oxford) 119 (2020) 109059.

[18] J. Deutscher, N. Gehring, R. Kern, Output feedback control of general linear heterodirectional hyperbolic ode--pde-ode systems, Automatica 95 (2018) 472-480.

[19] D. Bou Saba, F. Bribiesca-Argomedo, M. Di Loreto, D. Eberard, Strictly proper control design for the stabilization of $2 \times 2$ lin-810 ear hyperbolic ODE-PDE-ODE systems, in: Proceedings of the 58th IEEE Conference on Decision and Control, Nice, France, 2019.

[20] N. Gehring, A systematic design of backstepping-based state feedback controllers for ODE-PDE-ODE systems.

[21] H. Anfinsen, O. M. Aamo, Stabilization of a linear hyperbolic pde with actuator and sensor dynamics, Automatica 95 (2018) $104-111$

[22] U. Aarsnes, R. Vazquez, F. Di Meglio, M. Krstic, Delay robust control design of under-actuated PDE-ODE-PDE systems, in:820 American and Control Conference, 2019, pp. 3200-3205.

[23] J. Auriol, U. J. Aarsnes, F. Di Meglio, R. Shor, Robust control design of underactuated $2 \times 2$ PDE-ODE-PDE systems, IEEE control systems letters 5 (2) (2021) 469-474.

[24] J. Auriol, F. Bribiesca-Argomedo, Delay-robust stabilization of 825 an $n+m$ hyperbolic PDE-ODE system, in: IEEE Conference on Decision and Control and European Control Conference, 2019, pp. 4964-4970.

[25] J. Auriol, F. Bribiesca-Argomedo, S.-I. Niculescu, J. Redaud, Stabilization of a hyperbolic PDEs-ODE network using a recur-830 sive dynamics interconnection framework, in: IEEE European Control Conference (ECC), 2021 - under revision.

[26] I. Karafyllis, M. Krstic, Predictor Feedback for Delay Systems: Implementations and Approximations, Springer International Publishing, 2017.

[27] J. Auriol, F. Bribiesca Argomedo, D. Bou Saba, M. Di Loreto, F. Di Meglio, Delay-robust stabilization of a hyperbolic PDEODE system, Automatica 95 (2018) 494-502.

[28] G. Bastin, J.-M. Coron, Stability and boundary stabilization of 1-D hyperbolic systems, Springer, 2016.

[29] T. Meurer, A. Kugi, Tracking control for boundary controlled parabolic pdes with varying parameters: Combining backstepping and differential flatness, Automatica 45 (5) (2009) 11821194.

[30] I. Karafyllis, M. Krstic, Predictor feedback for delay systems: Implementations and approximations, Springer, 2017.

[31] H. Logemann, R. Rebarber, G. Weiss, Conditions for robustness and nonrobustness of the stability of feedback systems with re- spect to small delays in the feedback loop, SIAM Journal on Control and Optimization 34 (2) (1996) 572-600.

[32] J. Auriol, F. Di Meglio, An explicit mapping from linear first order hyperbolic PDEs to difference systems, Systems \& Control Letters 123 (2019) 144-150.

[33] J. Hale, S. Verduyn Lunel, Introduction to functional differential equations, Springer-Verlag, 1993.

[34] W. Michiels, S.-I. Niculescu, Stability and stabilization of timedelay systems: an eigenvalue-based approach, SIAM, 2007.

35] R. Vazquez, J.-M. Coron, M. Krstic, G. Bastin, Local exponential $H^{2}$ stabilization of a $2 \times 2$ quasilinear hyperbolic system using backstepping, in: Decision and Control and European Control Conference (CDC-ECC), 2011 50th IEEE Conference on, IEEE, 2011, pp. 1329-1334.

[36] K. Yoshida, Lectures on differential and integral equations, Vol. 10, Interscience Publishers, 1960.

37] D. Bresch-Pietri, F. Di Meglio, Prediction-based control of linear input-delay system subject to state-dependent state delayapplication to suppression of mechanical vibrations in drilling, Proc. of the 2nd IFAC Workshop on Control of Systems Governed by Partial Differential Equations 49 (8) (2016) 111-117.

[38] P.-O. Lamare, J. Auriol, F. Di Meglio, U. Aarsnes, Robust output regulation of $2 \times 2$ hyperbolic systems: Control law and input-to-state stability, in: American and Control Conference, 2018, pp. 1732-1739.

[39] J. Auriol, F. Bribiesca-Argomedo, D. Bresch-Pietri, Stabilization of an underactuated $1+2$ linear hyperbolic system with a proper control, in: Conference on Decision and Control, 2020.

[40] J. Auriol, F. Di Meglio, Minimum time control of heterodirectional linear coupled hyperbolic PDEs, Automatica 71 (2016) 300-307.

[41] R. J. LeVeque, Finite volume methods for hyperbolic problems, Cambridge university press, 2002.

[42] U. J. F. Aarsnes, N. van de Wouw, Axial and torsional selfexcited vibrations of a distributed drill-string, Journal of Sound and Vibration 444 (2019) 127-151.

[43] C. Germay, V. Denoël, E. Detournay, Multiple mode analysis of the self-excited vibrations of rotary drilling systems, Journal of Sound and Vibration 325 (1-2) (2009) 362-381. doi:10.1016/j.jsv.2009.03.017

II) URL http://linkinghub.elsevier.com/retrieve/pii/ S0022460X09002478

[44] U. J. F. Aarsnes, O. M. Aamo, Linear stability analysis of self-excited vibrations in drilling using an infinite dimensional model, Journal of Sound and Vibration 360 (2016) 239-259. doi:10.1016/j.jsv.2015.09.017

URL http://linkinghub.elsevier.com/retrieve/pii/ S0022460X15007385

545] E. Cayeux, On the Importance of Boundary Conditions for Real-Time Transient Drill-String Mechanical Estimations, in: IADC/SPE Drilling Conference and Exhibition, Society of Petroleum Engineers, 2018. doi:10.2118/189642-MS URL http://www . onepetro.org/doi/10.2118/189642-MS

[46] U. J. F. Aarsnes, O. M. Aamo, M. Krstic, Extremum seeking for real-time optimal drilling control, in: 2019 American Control Conference (ACC), IEEE, 2019, pp. 5222-5227.

[47] F. Di Meglio, U. J. F. Aarsnes, A distributed parameter systems view of control problems in drilling, IFAC-PapersOnLine 48 (6) (2015) 272-278. 